H. Sato

Nagoya Math. J.

Vol. 88 (1982), 79-119

\title{
ON AUGMENTED SCHOTTKY SPACES AND AUTOMORPHIC FORMS, II
}

\author{
HIROKI SATO
}

\section{Introduction}

This is the second half of the paper entitled "On augmented Schottky spaces and automorphic forms". In the first half, we introduced new coordinates to the Schottky space, and defined the augmented Schottky space $\hat{\subseteq}_{g}^{*}(\Sigma)$ by using the new coordinates. Furthermore we considered relations between the augmented Schottky space and Riemann surfaces with or without nodes.

In [2], Bers attached to each point $\tau$ of $\mathfrak{S}_{g}^{*} \backslash Z$ a basis of regular $q$ differentials on the Riemann surface $S(\tau)$, which depends holomorphically on $\tau$, where $\mathfrak{S}_{g}^{*}$ is the augmented Schottky space in the sense of Bers and $Z$ is a "small" set. Here we will establish the same results as in [2] for the case of the augmented Schottky space $\hat{S}_{g}^{*}(\Sigma)$ defined in the first half [4]; Theorem 2 is the main theorem.

In $\S 1$, we will state some notations and quote a known proposition needed later. In $\S 2$, we will construct four kinds of Poincaré series and in $\S 3$, we will consider their properties. In $\S 4$, we will consider continuity of the functions constructed in $\S 2$. Proposition 6 is decomposed into Propositions 7 and 8 which will be proved in the next section, $\S 5$. The technique of the proofs of Propositions 7 and 8 plays an important role in the proofs of the later propositions. In $\S 6$, we will again consider continuity of the functions. We summarize from Proposition 5 through Proposition 12 in Proposition 13. Proposition 14 says that the functions constructed in $\S 2$ are holomorphic on the fiber spaces over the augmented Schottky spaces (see [5] for the definition of the fiber space). In $\S 7$, we will consider four propositions which guarantee that $(2 q-1)(g-1)$ linear combinations of the functions form a basis of regular $q$-differentials on the Riemann surfaces $S(\tau)$ for each $\tau \in \hat{S}_{g}^{*}(\Sigma) \backslash Z$, where $Z$ is a "small" set. In $\S 8$, we

Received May 31, 1980. 
will state three main theorems. We will use the same notations and terminologies as in Sato [4] and [5].

This paper was written while the author stays at the State University of New York at Stony Brook. He wishes to express his deepest gratitude to Professors I. Kra, B. Maskit and P. Matelski for many advices and suggestions.

\section{§1. Definitions and Preliminary}

Definition. A holomorphic $q$-differential ( $q>0$; an integer) on a compact Riemann surface without nodes is a holomorphic form of type $(q, 0)$. In other word, it is locally represented as $F=f(z) d z^{q}$, where $z$ is a local parameter and $f$ is a holomorphic function.

Let $p \in S$. Let $z$ be a local parameter with $z=0$ at $p$ defined on $D$ $(p \in D \subset S)$. Then a holomorphic $q$-differential on $D \backslash\{p\}$ is written as $F$ $=\sum_{n=-\infty}^{+\infty} a_{n} z^{n} d z^{q}$ in a neighborhood of $p$. The coefficient $a_{-q}$ is called the residue of $F$ at $p$.

Definition. A regular q-differential on a stable compact Riemann surface $S$ with nodes (for the definition see Bers [2], p. 344) is a holomorphic $q$-differential on each part of $S$ satisfying the following properties:

(i) It has poles of order at most $q$ at the punctures corresponding to nodes.

(ii) Let $a_{-q}$ and $a_{-q}^{*}$ be the residues at two punctures joining the nodes. Then $a_{-q}=a_{-q}^{*}$ if $q$ is even and $a_{-q}=-a_{-q}^{*}$ if $q$ is odd.

Let $S$ be a compact Riemann surface of genus $g$ with or without nodes. Let $d=d(q, g)$ be a number of linearly independent regular $q$-differentials on $S$. Then by the Riemann-Roch theorem,

$$
d=\left\{\begin{array}{ll}
q & \text { if } q=1 \\
(2 q-1)(g-1) & \text { if } q>1
\end{array} .\right.
$$

Let $G$ be a Kleinian group and let $\Omega(G)$ be the region of discontinuity of $G$. Let $\Omega^{\prime}(G)$ be a subset of $\Omega(G)$ with the following properties: (i) $\gamma\left(\Omega^{\prime}(G)\right)=\Omega^{\prime}(G)$ for all $\gamma \in G$ and (ii) $\Omega(G) \backslash \Omega^{\prime}(G)$ is a discrete set.

Definition. Let $\phi(z), z \in \Omega^{\prime}(G)$, be a holomorphic function with $\phi(A z) A^{\prime}(z)^{q}=\phi(z)$ for all $A \in G$. Then $\phi(z)$ is called a holomorphic form of weight $(-2 q)$ on $\Omega^{\prime}(G)$. For simplicity it is called a holomorphic $q$-form.

This form induces a holomorphic $q$-differential on each part of $S(G)$ 
$=\Omega(G) / G$. Let $G_{0}$ be a subgroup of $G$. Let $\gamma_{0}, \gamma_{1}, \gamma_{2}, \cdots$ be a complete list of the right coset representatives of $G$ modulo $G_{0}$ as $G=G_{0} r_{0}+G_{0} r_{1}$ $+G_{0 \gamma_{2}}+\cdots$. Let $\Phi(z)$ be a holomorphic function with $\Phi(\gamma(z)) \gamma^{\prime}(z)^{q}=\Phi(z)$ for all $\gamma \in G_{0}$. Set $\phi(z)=\sum_{n=0}^{\infty} \Phi\left(\gamma_{n}(z)\right) \gamma_{n}^{\prime}(z)^{q}$. If the series converges uniformly on any compact subset, then $\phi$ is a holomorphic $q$-form for $G$.

Proposition 1 (Bers [2]). Let $\Delta$ be a domain in $C^{r}$ and let $\Gamma$ be a properly discontinuous group of holomorphic self-mappings of $\Delta$ :

$$
\Delta \ni \zeta=\left(\zeta^{1}, \cdots, \zeta^{r}\right) \longrightarrow \gamma(\zeta)=\left(\gamma^{1}(\zeta), \cdots, \gamma^{r}(\zeta)\right) \in \Delta .
$$

Let $\Gamma_{0}$ be a subgroup and let $\gamma_{0}, \gamma_{1}, \gamma_{2}, \ldots$ be a complete list of the right coset representatives of $\Gamma$ modulo $\Gamma_{0}$. Let $q \geqq 1$ be an integer. Let $\rho(\zeta)$, $\zeta \in \Delta$, be a positive continuous function such that $\rho(\gamma(\zeta)) \mid$ jac $_{r}(\zeta) \mid=\rho(\zeta), \gamma \in \Gamma$, where $\operatorname{jac}_{r}(\zeta)=\partial\left(\gamma^{1}, \cdots, \gamma^{r}\right) / \partial\left(\zeta^{1}, \cdots, \zeta^{r}\right)$. Let $\Phi(\zeta), \zeta \in \Delta$, be a holomorphic function such that $\Phi(\gamma(\zeta))$ jac $_{r}(\zeta)^{q}=\Phi(\zeta), \gamma \in \Gamma_{0}$ and

$$
\iint_{\Delta / \Gamma_{0}} \rho(\zeta)^{2-q}|\Phi(\zeta)| d V_{\zeta}<+\infty
$$

where $d V_{\zeta}=d \xi^{1} d \eta^{1} \cdots d \xi^{r} d \eta^{r}\left(\zeta^{j}=\zeta^{j}+\sqrt{-1} \eta^{j}\right)$. Set

$$
\phi(\zeta)=\sum_{n=0}^{\infty} \Phi\left(\gamma_{n}(\xi)\right) \mathrm{jac}_{\gamma_{n}}(\zeta)^{q}, \quad \zeta \in \Delta .
$$

Then

(i) this series converges absolutely and uniformly on any compact subset of $\Delta$,

(ii) $\phi(\zeta)$ is holomorphic in $\Delta$,

(iii) $\phi(\gamma(\zeta))$ jac $_{r}(\zeta)^{q}=\phi(\zeta)$ for all $\gamma \in \Gamma$, and

(iv) $\iint_{\Delta / \Gamma} \rho(\zeta)^{2-q}|\phi(\zeta)| d V_{\zeta} \leqq \iint_{\Delta / \Gamma_{0}} \rho(\zeta)^{2-q}|\Phi(\zeta)| d V_{\zeta}$.

\section{§2. Construction of Poincaré theta series}

From now on, we fix integers $g \geqq 2$ and $q \geqq 2$. We fix a compact Riemann surface $S$ of genus $g$ and a standard system of loops $\Sigma=\left\{\alpha_{1}, \cdots\right.$, $\left.\alpha_{g} ; \gamma_{1}, \cdots, \gamma_{2 g-3}\right\}$ on $S$ (see [4] for the definition). For the definitions of fiber spaces $\widetilde{\mathscr{V}}_{s} \hat{\Theta}_{g}^{*}(\Sigma)$ and Schottky groups $\tilde{G}_{s}(\tau)$, see [5]. Here we shall define four kinds of functions of $3 g-2$ variables.

2-1. Let $(\tau, z) \in \widetilde{F}_{s} \delta^{I, J} \widetilde{S}_{g}(\Sigma)$. Set $\tilde{S}_{s}(\tau)=\Omega\left(\tilde{G}_{s}(\tau)\right) \backslash \tilde{G}_{s}(\tau)(s=0,1, \ldots$, $2 g-3)$. There are two kinds of distinguished points on $\tilde{S}_{s}(\tau)$. One is the following: To every $i \in I$, there correspond distinguished points $\hat{p}_{i}(\tau)$ and 
$\hat{q}_{i}(\tau) \in S(\tau)$ (see [4], p. 172 and [5], p. 74). We call them the distinguished points of the first kind. We denote by $I_{s}$ the totality of $i$ such that $\hat{p}_{i}(\tau)$, $\hat{q}_{i}(\tau) \in \widetilde{S}_{s}(\tau)$.

The other is the right and the left distinguished points $\hat{p}_{j}^{+}(\tau)$ and $\hat{p}_{j}^{-}(\tau)$ $\in S(\tau)$ corresponding to every $j \in J$ (see [4], p. 172). We call them the distinguished points of the second kind. We denote by $J_{s}$ the totality of $j$ such that either $\hat{p}_{j}^{+}(\tau)$ or $\hat{p}_{j}^{-}(\tau)$ belongs to $\tilde{S}_{s}(\tau)$.

Let $A_{s, 1}(\tau, z), \cdots, A_{s, g_{s}}(\tau, z)$ be generators of $\tilde{G}_{s}(\tau)$. We take defining curves $C_{s, 1}(\tau), C_{s, 1}^{\prime}(\tau), \cdots, C_{s, g_{s}}(\tau), C_{s, g_{s}}^{\prime}(\tau)$ of $\tilde{G}_{s}(\tau)$. Let $\omega_{s}(\tau)$ be the standard fundamental domain for $\tilde{G}_{s}(\tau)$ bounded by them. Let $p_{s, h}(\tau)$ and $q_{s, h}(\tau)$ be the repelling and the attracting fixed points of $A_{s, h}(\tau, z)\left(h=1,2, \cdots, g_{s}\right)$. We denote by $P_{s, 1}(\tau)$ the set of these points.

Let $I_{s}=\left\{i_{s, g_{s}+1}, \cdots, i_{s, g_{s+k_{s}}}\right\}, i_{s, g_{s+1}}<\cdots<i_{s, g_{s}+k_{s}}$. For simplicity we write $\hat{p}_{s, g_{s+h}}(\tau)$ and $\hat{q}_{s, g_{s+h}}(\tau)$ instead of $p_{i}(\tau)$ and $q_{t}(\tau)$, respectively, for $i=$ $i_{s, g_{s+h}}$. Let $p_{s, g_{s}+h}(\tau)$ and $q_{s, g_{s+h}}(\tau)$ be the lifts of $\hat{p}_{s, g_{s+h}}(\tau)$ and $\hat{q}_{s, g_{s}+h}(\tau)$ to $\omega_{s}(\tau)$, respectively. We also call them the distinguished points of the first kind. We denote by $P_{s, 2}(\tau)$ the set of these points.

Let $J_{s}=\left\{j_{s, 1}, \cdots, j_{s, n_{s}}\right\}, j_{s, 1}<\cdots<j_{s, n_{s}}$. For simplicity we write $\hat{p}_{s, k}^{ \pm}(\tau)$ instead of $\hat{p}_{j, k}^{ \pm}(\tau)$. Let $p_{s, k}^{ \pm}(\tau)$ be the lifts of $\hat{p}_{s, k}^{ \pm}(\tau)$ to $\omega_{s}(\tau)$. We also call them the distinguished points of the second kind. We denote by $P_{s, 3}(\tau)$ the set of these points. We put $P_{s}(\tau)=P_{s, 1}(\tau) \cup P_{s, 2}(\tau) \cup P_{s, 3}(\tau)$.

2-2. Now we will construct four kinds of functions. We define $\Phi_{s, h}(\tau, z)$ by setting

$$
\Phi_{s, h}(\tau, z)=\left\{\frac{p_{s, h}(\tau)-q_{s, h}(\tau)}{\left(z-p_{s, h}(\tau)\right)\left(z-q_{s, h}(\tau)\right)}\right\}^{q},
$$

for $(\tau, z) \in \widetilde{\mathfrak{V}}_{s} \delta^{I, J} \widetilde{S}_{g}(\Sigma)\left(s=0,1, \cdots, 2 g-3 ; h=1,2, \cdots, g_{s}+k_{s}\right)$. In particular, if $p_{s, h}(\tau)=0$ and $q_{s, h}(\tau)=\infty$, then we define $\Phi_{s, h}(\tau, z)=1 / z^{q}$. We denote by $N_{s}(\tau)$ the set of all linear combinations of $\Phi_{s, h}(\tau, z)\left(h=1,2, \cdots, g_{s}+k_{s}\right)$. For $h=1,2, \cdots, g_{s}$, let $\hat{G}_{s, h}(\tau)$ be the group generated by $A_{s, h}(\tau, z)$, that is, $\hat{G}_{s, h}(\tau)=\left\langle A_{s, h}(\tau, z)\right\rangle$. For $h=g_{s}+1, \cdots, g_{s}+k_{s}$, put $\hat{G}_{s, h}(\tau)=\{1\}$. Let $\gamma_{0}, \gamma_{1}, \gamma_{2}, \cdots$ be a complete list of the right coset representatives of $\tilde{G}_{s}(\tau)$ modulo $\hat{G}_{s, h}(\tau)$. We set

$$
\begin{aligned}
\phi_{s, h}(\tau, z)= & \sum_{n=0}^{\infty} \Phi_{s, h}\left(\tau, \gamma_{n}(z)\right) \gamma_{n}^{\prime}(z)^{q} \\
& (\tau, z) \in \widetilde{\mho}_{s} \delta^{I, J} \widetilde{S}_{g}(\Sigma) \\
& \left(s=0,1,2, \cdots, 2 g-3 ; h=1,2, \cdots, g_{s}+k_{s}\right) .
\end{aligned}
$$


2-3. We denote by $M_{s}(\tau)$ the space of all the meromorphic functions in $z$ having the following properties:

(i) They have poles of order at most $q-1$ at

$$
\begin{aligned}
& p_{s, 1}(\tau), \quad q_{s, 1}(\tau), \cdots, p_{s, g_{s}+k_{s}}(\tau), \quad q_{s, g_{s}+k_{s}}(\tau), \\
& p_{s, 1}^{\prime}(\tau), \cdots, p_{s, n_{s}}^{\prime}(\tau),
\end{aligned}
$$

where $p_{s, k}^{\prime}(\tau)$ represents either $p_{s, k}^{+}(\tau)$ or $p_{s, k}^{-}(\tau)$ whose projection belongs to $\tilde{S}_{s}(\tau)$.

(ii) They have no other singularities.

(iii) If $\infty$ does (resp. does not) appear among the points (3), then they have zero of order at least $q+1$ (resp. $2 q$ ) at $\infty$.

We denote by $\Pi_{n}$ the space of polynomials of degree at most $n$. If $\infty$ does not appear among the points (3), an element of $M_{s}(\tau)$ is represented by

$$
\Theta_{s, \pi}(\tau, z)=\frac{\pi(z)}{\left\{\left(z-p_{s, 1}(\tau)\right)\left(z-q_{s, 1}(\tau)\right) \cdots\left(z-p_{s, n_{s}}^{\prime}(\tau)\right)\right\}^{q-1}}
$$

with $\pi \in \Pi_{e_{s}-1}$, where $e_{s}=\left\{2\left(g_{s}+k_{s}\right)+n_{s}-2\right\} q-\left\{2\left(g_{s}+k_{s}\right)+n_{s}\right\}+1$. Therefore $\operatorname{dim} M_{s}(\tau)=e_{s}$. In the case where the sequence of points (3) contains $\infty, \Theta_{s, \pi}(\tau, z)$ is similarly defined and we also obtain $\operatorname{dim} M_{s}(\tau)=e_{s}$.

We set

$$
\begin{aligned}
& \theta_{s, \pi}(\tau, z)=\sum_{r \in \widetilde{G}_{s}(\tau)} \Theta_{s, \pi}(\tau, \gamma(z)) \gamma^{\prime}(z)^{q} \\
& \quad(\tau, z) \in \widetilde{\mho}_{s} \delta^{I, J} \widetilde{S}_{g}(\Sigma), \quad s=0,1,2, \cdots, 2 g-3 .
\end{aligned}
$$

2-4. Here we define $\Psi_{s, k}(\tau, z)\left(s=0,1,2, \cdots, 2 g-3 ; k=1,2, \cdots, n_{s}\right)$ in the case where the sequence (3) does not contain $\infty$. We set, if $g_{s}+k_{s} \geqq 1$,

$$
\Psi_{s, k}(\tau, z)=\frac{1}{\left(z-p_{s, k}^{\prime}(\tau)\right)^{q}\left(z-p_{s, 1}(\tau)\right)^{q-1}\left(z-q_{s, 1}(\tau)\right)}
$$

if $g_{s}+k_{s}=0$

$$
\left\{\begin{aligned}
& \Psi_{s, 1}(\tau, z)=\frac{1}{\left(z-p_{s, 1}^{\prime}(\tau)\right)^{q}\left(z-p_{s, 2}^{\prime}(\tau)\right)^{q-1}\left(z-p_{s, 3}^{\prime}(\tau)\right)} \\
& \Psi_{s, 2}(\tau, z)=\frac{1}{\left(z-p_{s, 2}^{\prime}(\tau)\right)^{q}\left(z-p_{s, 1}^{\prime}(\tau)\right)^{q-1}\left(z-p_{s, 3}^{\prime}(\tau)\right)} \\
& \Psi_{s, k}(\tau, z)=\frac{1}{\left(z-p_{s, k}^{\prime}(\tau)\right)^{q}\left(z-p_{s, 1}^{\prime}(\tau)\right)^{q-1}\left(z-p_{s, 2}^{\prime}(\tau)\right)} \\
&\left(k=3,4, \cdots, n_{s}\right)
\end{aligned}\right.
$$


They are similarly defined in the case where the sequence (3) contains $\infty$. The detail is left for the reader.

We denote by $L_{s}(\tau)$ the set of all the linear combinations of elements $\Psi_{s, k}(\tau, z)\left(k=1,2, \cdots, n_{s}\right)$ and elements of $M_{s}(\tau)$ which do not belong to $M_{s}(\tau)$. We set

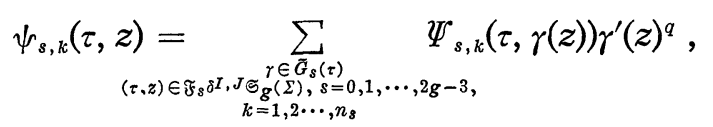

2-5. In order to introduce the fourth functions we need some preparation. We denote by $G_{s}^{I, J}$ the abstract free group generated by $A_{1}, A_{2}, \cdots$, $A_{g_{s}}$. For $\tau^{\prime} \in \mathbb{S}_{g}^{I^{\prime}, J^{\prime}}(\Sigma)$ with $I^{\prime} \subset I$ and $J^{\prime} \subset J$, we let $\hat{G}_{s}\left(\tau^{\prime}\right)$ be the group generated by $A_{s, 1}\left(\tau^{\prime}, z\right), \cdots, A_{s, g_{s}}\left(\tau^{\prime}, z\right)$. Denote by $\eta_{\tau}$, the canonical isomorphism of $G_{s}^{I, J}$ onto $\hat{G}_{s}\left(\tau^{\prime}\right)$ defined by the correspondence $A_{h} \mapsto A_{s, h}\left(\tau^{\prime}, z\right)$. We note that $\hat{G}_{s}\left(\tau^{\prime}\right)$ is a Schottky group and a subgroup of $\tilde{G}_{s}\left(\tau^{\prime}\right)$. The image of $\gamma \in G_{s}^{I, J}$ under the isomorphsm $\eta_{\tau^{\prime}}$, will be denoted by $z \mapsto \gamma\left(\tau^{\prime}, z\right)$. Assume $\infty \notin \Omega^{\prime}\left(\tilde{G}_{s}(\tau)\right)$. Let $\mathscr{S}_{s}^{I, J}$ denote the set of all sequences $\sigma$ of $2 q-1$ distinct elements $\gamma_{1}, \cdots, \gamma_{2 q-1}$ of $G_{s}^{I, J}$ with the property: The attracting fixed points $a_{1}\left(\tau^{\prime}\right), \cdots, a_{2 q-1}\left(\tau^{\prime}\right)$ of $\gamma_{1}\left(\tau^{\prime}, z\right), \cdots, \gamma_{2 q-1}\left(\tau^{\prime}, z\right)$ are distinct for one (and hence for all) $\tau^{\prime} \in \mathbb{S}_{g}^{I, J}(\Sigma)$. For $T \tilde{G}_{s}\left(\tau^{\prime}\right) T^{-1}$ with $T \in$ Möb such that $\infty \in \Omega^{\prime}\left(T \tilde{G}_{s}\left(\tau^{\prime}\right) T^{-1}\right)$, let $\mathscr{S}_{s}^{I, J}$ denote the set of all sequences $\sigma$ of $2 q$ distinct elements $\gamma_{1}, \cdots, \gamma_{2 q}$ of $G_{s}^{I, J}$ with the same property as above.

Now we define the functions $\Psi_{s, \sigma}(\tau, z)$ as follows. Let $\sigma \in \mathscr{S}_{s}^{I, J}$ and $(\tau, z) \in \widetilde{\mho}_{s} \widetilde{S}_{g}^{I, J}(\Sigma)$. If $\infty \notin \Omega^{\prime}\left(\tilde{G}_{s}(\tau)\right)$, then we set

$$
\Psi_{s, \sigma}(\tau, z)=\frac{1}{\left(z-a_{1}(\tau)\right)\left(z-a_{2}(\tau)\right) \cdots\left(z-a_{2 q-1}(\tau)\right)}
$$

and

$$
\psi_{s, \sigma}(\tau, z)=\sum_{\gamma \in \tilde{G}_{s}(\tau)} \Psi_{s, \sigma}(\tau, \gamma(z)) \gamma^{\prime}(z)^{q}
$$

If $\infty \in \Omega^{\prime}\left(\tilde{G}_{s}(\tau)\right)$, then we set

$$
\Psi_{s, \sigma}(\tau, z)=\frac{1}{\left(z-a_{1}(\tau)\right)\left(z-a_{2}(\tau)\right) \cdots\left(z-a_{2 q}(\tau)\right)} .
$$

$\psi_{s, \sigma}(\tau, z)$ is defined by (9).

2-6. Let $\tau \in \hat{\Im}_{g}^{*}(\Sigma), z \in \Omega^{\prime}\left(\tilde{G}_{s}(\tau)\right)$ and $w \in \Omega^{\prime}\left(\tilde{G}_{t}(\tau)\right)$. Suppose that there exists $T \in$ Möb such that $\tilde{G}_{t}(\tau)=T \tilde{G}_{s}(\tau) T^{-1}$. Then we call $\tilde{G}_{t}(\tau)$ being 
equivalent to $\tilde{G}_{s}(\tau)$. Let $\phi_{s}$ be an automorphic form of weight $(-2 q)$ on $\Omega^{\prime}\left(\tilde{G}_{s}(\tau)\right)$ for $\tilde{G}_{s}(\tau)$. We define $\phi_{t}$ by setting $\phi_{t}(w)=\phi_{s}\left(T^{-1}(w)\right) T^{-1^{\prime}}(w)^{q}$. Then $\phi_{t}(w)$ is an automorphic form of weight $(-2 q)$ on $\Omega^{\prime}\left(\tilde{G}_{t}(\tau)\right)$ for $\tilde{G}_{t}(\tau)$. We say $\phi_{t}$ being equivalent to $\phi_{s}$.

We easily see the following: If $\Phi_{s, h} \in N_{s}(\tau)$, then $\Phi(\tau, w)$ defined by $\Phi(\tau, w)=\Phi_{s, h}\left(\tau, T^{-1}(w)\right) T^{-1^{\prime}}(w)^{q}$ belongs to $N_{t}(\tau)$. Furthermore $\phi(\tau, w)=$ $\phi_{s, h}\left(\tau, T^{-1}(w)\right) T^{-1^{\prime}}(w)^{q}$, namely $\phi(\tau, w)$ is equivalent to $\phi_{s, h}(\tau, z)$, where $\phi(\tau, w)$ is the Poincaré theta series of $\Phi(\tau, w)$ for $\tilde{G}_{t}(\tau)$. For $\theta_{s, \pi}, \psi_{s, k}$ and $\psi_{s, a}$, the same holds.

So far we defined $\phi_{s, h}(\tau, z), \theta_{s, \pi}(\tau, z), \psi_{s, k}(\tau, z)$, and $\psi_{s, \sigma}(\tau, z)$ for $(\tau, z) \in$ $\widetilde{\mho}_{s} \hat{\Im}_{g}^{*}(\Sigma)$. Now we extend them to $(\tau, z)$ in the union $\sum_{t=0}^{2 g-3} \widetilde{\mho}_{t} \hat{\Im}_{g}^{*}(\Sigma)$.

For this purpose, let $\tau \in \delta^{I, J} \widetilde{S}_{g}(\Sigma)$ and $z \in \Omega^{\prime}\left(\widetilde{G}_{t}(\tau)\right)$. If the cells $\sigma_{s}$ and $\sigma_{t}$ belong to the same part $\left[\sigma_{j_{k}}\right]$ (i.e., $k(s)=k(t)$, see Remark in [5], p. 75), then there exists $T \in$ Möb such that $\tilde{G}_{t}(\tau)=T \tilde{G}_{s}(\tau) T^{-1}$ (see [5], p. 75). In this case, we put $\phi_{s, h}(\tau, z)=\phi_{s, h}\left(\tau, T^{-1}(z)\right) T^{-1^{\prime}}(z)^{q}$ for $z \in \Omega^{\prime}\left(\tilde{G}_{t}(\tau)\right)$. If $s$ and $t$ do not have the above relation, then put $\phi_{s, h}(\tau, z)=0$ for $z \in \Omega^{\prime}\left(\tilde{G}_{t}(\tau)\right)$.

We extend the functions $\theta_{s, \pi}(\tau, z)$ and $\psi_{s, o}(\tau, z)$ in completely the same way.

Next, with respect to every $j \in J$, we introduce the function $\psi_{j}(\tau, z)$ defined on $\sum_{t=0}^{2 g-3} \widetilde{\mho}_{t} \hat{\Im}_{g}^{*}(\Sigma)$. For $\tau \in \delta^{I, J} \widetilde{\subseteq}_{g}(\Sigma)$ and $z \in \Omega\left(\tilde{G}_{t}(\tau)\right)$, we put $\psi_{j}(\tau, z)$ $=0$ if $j \oplus J_{t}$, and $\psi_{j}(\tau, z)=\psi_{t, k}(\tau, z)$ if $j \in J_{t}=\left\{j_{t, 1}, \cdots, j_{t, n_{t}}\right\}$ and $j=j_{t, k}$ (remark: $j \in J_{t}$ if and only if either $p_{j}^{+}(\tau)$ or $p_{j}^{-}(\tau)$ belongs to $S_{k(t)}$; for the notation $k(t)$, see [5], p. 74).

§3. Properties of functions $\Phi_{s, h}(\tau, z), \Theta_{s, \pi}(\tau, z), \Psi_{s, k}(\tau, z)$ and $\Psi_{s, \sigma}(\tau, z)$

3-1. We fix a compact Riemann surface $S$ of genus $g$ and a standard system of loops $\Sigma=\left\{\alpha_{1}, \cdots, \alpha_{g} ; \gamma_{1}, \cdots, \gamma_{2 g-3}\right\}$ on $S$. Let $I=\left\{i_{1}, \cdots, i_{k}\right\}$ and $J=\left\{j_{1}, \cdots, j_{m}\right\}$. We will consider the following deformation

$$
\lim _{\nu \rightarrow \infty} \tau_{\nu}=\tau_{0},
$$

where $\tau_{\nu} \in \delta^{I, J \widetilde{S}_{g}}(\Sigma)$ and $\tau_{0} \in \delta^{I, L \widetilde{S}_{g}}(\Sigma)$ with $L=J \cup\{l\} \subset\{1,2, \cdots, 2 g-3\}$. By the dividing loops $\gamma_{j_{1}}, \cdots, \gamma_{j_{m}}, S$ is divided into $m+1$ parts $\left[\sigma_{0}\right]=\left[\sigma_{0}(J)\right]$, $\left[\sigma_{j_{1}}\right]=\left[\sigma_{j_{1}}(J)\right], \cdots,\left[\sigma_{j_{m}}\right]=\left[\sigma_{j_{m}}(J)\right]$. Since $l$ is not contained in $J, \gamma_{l}$ lies on $\left[\sigma_{r}(J)\right]$ for some $r \in\left\{0, j_{1}, \cdots, j_{m}\right\}$. Then $\gamma_{l}$ divides $\left[\sigma_{r}(J)\right]$ into two parts $\left[\sigma_{r}(L)\right]$ and $\left[\sigma_{l}(L)\right]$. Corresponding to $\tau_{\nu}$, there are $m+1$ Schottky groups (including the trivial group) $G_{0}\left(\tau_{\nu}\right), G_{j_{1}}\left(\tau_{\nu}\right), \ldots, G_{j_{m}}\left(\tau_{\nu}\right)$ and $m+1$ Riemann surfaces $S_{0}\left(\tau_{\nu}\right), S_{j_{1}}\left(\tau_{\nu}\right), \cdots, S_{j_{m}}\left(\tau_{\nu}\right)$. Similarly corresponding to $\tau_{0}$, there are 
$m+2$ Schottky groups $G_{r}\left(\tau_{0}\right), G_{l}\left(\tau_{0}\right)$ and $G_{j_{k}}\left(\tau_{0}\right)\left(j_{k} \in J \cup\{0\} \mid\{r\}\right)$ and $m+2$ Riemann surfaces $S_{r}\left(\tau_{0}\right), S_{l}\left(\tau_{0}\right)$ and $S_{j_{k}}\left(\tau_{0}\right)\left(j_{k} \in J \cup\{0\} \backslash\{r\}\right)$.

We use the same notations and terminologies as in $\S 2-1$. We remark that $G_{j_{k}}\left(\tau_{0}\right)=\tilde{G}_{j_{k}}\left(\tau_{0}\right) \quad\left(j_{k} \in J \cup\{0\} \backslash\{r\}\right), \quad G_{r}\left(\tau_{0}\right)=\tilde{G}_{r}\left(\tau_{0}\right)$ and $G_{l}\left(\tau_{0}\right)=\tilde{G}_{l}\left(\tau_{0}\right)$. Furthermore $G_{j_{k}}\left(\tau_{\nu}\right)=\tilde{G}_{j_{k}}\left(\tau_{\nu}\right)\left(j_{k} \in J \cup\{0\}\right)$ and $\tilde{G}_{l}\left(\tau_{\nu}\right)=\tilde{T}_{\nu} \tilde{G}_{r}\left(\tau_{\nu}\right) \tilde{T}_{\nu}^{-1}$ for some $\tilde{T}_{\nu} \in$ Möb; remember that $r$ is determined by $\gamma_{l} \subset\left[\sigma_{r}(J)\right]$. Let $\tilde{G}_{r}\left(\tau_{0}\right)=$ $\left\langle A_{r, 1}\left(\tau_{0}, z\right), \cdots, A_{r, g_{r}}\left(\tau_{0}, z\right)\right\rangle$, and $\tilde{G}_{l}\left(\tau_{0}\right)=\left\langle A_{l, 1}^{*}\left(\tau_{0}, z\right), \cdots, A_{l, g_{l}}^{*}\left(\tau_{0}, z\right)\right.$. We set $\hat{G}_{r}\left(\tau_{\nu}\right)=\left\langle A_{r, 1}\left(\tau_{\nu}, z\right), \cdots, A_{r, g_{r}}\left(\tau_{\nu}, z\right)\right\rangle, \hat{G}_{l}^{*}\left(\tau_{\nu}\right)=\left\langle A_{l, 1}^{*}\left(\tau_{\nu}, z\right), \cdots, A_{l, g_{l}}^{*}\left(\tau_{\nu}, z\right)\right\rangle$ and $\hat{G}_{l}\left(\tau_{\nu}\right)=\tilde{T}_{\nu}^{-1} \hat{G}_{l}^{*}\left(\tau_{\nu}\right) \tilde{T}_{\nu}=\left\langle A_{l, 1}\left(\tau_{\nu}, z\right), \cdots, A_{l, g_{l}}\left(\tau_{\nu}, z\right)\right\rangle$, where

$$
A_{l, i}\left(\tau_{\nu}, z\right)=\tilde{T}_{\nu}^{-1} A_{l, i}^{*}\left(\tau_{\nu}, z\right) \tilde{T}_{\nu} \quad\left(i=1,2, \cdots, g_{l}\right) .
$$

Let $\gamma_{r}=\gamma\left(1, i_{1}, \cdots, i_{\mu(r)}\right)$ and $\gamma_{l}=\gamma_{l}=\gamma\left(1, i_{1}, \cdots, i_{\mu(l)}\right)$. We take standard fundamental domains $\omega_{r}\left(\tau_{\nu}\right), \omega_{r}\left(\tau_{0}\right), \omega_{l}\left(\tau_{\nu}\right)^{*}, \omega_{l}\left(\tau_{0}\right)^{*}$ for $\tilde{G}_{r}\left(\tau_{\nu}\right), \tilde{G}_{r}\left(\tau_{0}\right), \tilde{G}_{l}\left(\tau_{\nu}\right)$, and $\tilde{G}_{l}\left(\tau_{0}\right)$, respectively. We remark that the distinguished points $p_{l}^{+}\left(\tau_{0}\right)$ and $p_{l}^{-}\left(\tau_{0}\right)$ are in $\omega_{r}\left(\tau_{0}\right)$ and $\omega_{l}\left(\tau_{0}\right)^{*}$, respectively, and that for every large $\nu$, $p_{l}^{+}\left(\tau_{0}\right) \in \omega_{r}\left(\tau_{\nu}\right)$ and $p_{l}^{-}\left(\tau_{0}\right) \in \omega_{l}\left(\tau_{\nu}\right)^{*}$. We choose small circles $c_{r}$ (resp. $c_{l}^{*}$ ) with center at $p_{l}^{+}\left(\tau_{0}\right)$ (resp. $\left.p_{l}^{-}\left(\tau_{0}\right)\right)$ in $\omega_{r}\left(\tau_{0}\right)$ (resp. $\left.\omega_{l}\left(\tau_{0}\right)^{*}\right)$. It is possible to choose, for every large $\nu$, all the defining curves of $\hat{G}_{l}\left(\tau_{\nu}\right)$ (resp. $\left.\hat{G}_{r}^{*}\left(\tau_{\nu}\right)=\tilde{T}_{\nu} \hat{G}_{r}\left(\tau_{\nu}\right) \tilde{T}_{\nu}^{-1}\right)$ to be contained in the interior to $c_{r}$ (resp. $c_{l}^{*}$ ) and all the defining curves of $\hat{G}_{r}\left(\tau_{\nu}\right)$ (resp. $\hat{G}_{l}^{*}\left(\tau_{\nu}\right)$ ) to lie to the exterior to $c_{r}$ (resp. $c_{l}^{*}$ ) (see the proof of Proposition 5 in [4], pp. 169-170).

We take a small disk $D_{r}$ (resp. $\left.D_{l}^{*}\right)$ inside of $\omega_{r}\left(\tau_{0}\right) \cap \Omega^{\prime}\left(\tilde{G}_{r}\left(\tau_{0}\right)\right.$ ) (resp. $\omega_{l}\left(\tau_{0}\right)^{*} \cap \Omega^{\prime}\left(\tilde{G}_{l}\left(\tau_{0}\right)\right)$ and to the exterior to $c_{r}$ (resp. $c_{l}^{*}$ ). We denote by $d_{r}$ (resp. $d_{l}^{*}$ ) the center of $D_{r}$ (resp. $\left.D_{l}^{*}\right)$ and set $d_{r}^{*}\left(\tau_{\nu}\right)=\tilde{T}_{\nu}\left(d_{r}\right)$ and $d_{l}\left(\tau_{\nu}\right)=$ $\tilde{T}_{\nu}^{-1}\left(d_{l}\right)$.

We obtain a new group from $\tilde{G}_{r}\left(\tau_{\nu}\right)$ by the following normalization: (1) $p_{r}^{-}\left(\tau_{\nu}\right)=0$ (2) $d_{r}=\infty$, and (3) $p^{(+)}\left(1, i_{1}, \cdots, i_{\mu_{(l)}}, 0, \cdots, 0\right)\left(\tau_{\nu}\right)=1$, where $P^{(+)}\left(1, i_{1}, \cdots, i_{\mu(l)}, 0, \cdots, 0\right)\left(\tau_{\nu}\right)$ means an attracting or a repelling fixed point $p(1, i_{1}, \cdots, i_{\mu(l)}, \underbrace{0, \cdots, 0}_{m})\left(\tau_{\nu}\right)$ (see $\left([4]\right.$, p. 165) if $\sigma(1, i_{1}, \cdots, i_{\mu(l)}, \underbrace{0, \cdots, 0}_{m})$ is the terminal cell in $\left[\sigma_{r}(J)\right]$, and the right distinguished point $p^{+}\left(1, i_{1}, \cdots\right.$, $i_{\mu(l)}, \underbrace{0, \cdots, 0}_{m^{\prime}})\left(\tau_{\nu}\right)$ with respect to the boundary loop $\gamma(1, i_{1}, \cdots, i_{\mu}, \underbrace{0, \cdots, 0}_{m^{\prime}})$ of $\left[\sigma_{r}(J)\right]$ if $\sigma(1, i_{1}, \cdots, i_{\mu(l)}, \underbrace{0, \cdots, 0)}_{m} \oplus\left[\sigma_{r}(J)\right]$. We denote again by $\tilde{G}_{r}^{m^{\prime}}\left(\tau_{\nu}\right)$ the new group.

Next we obtain a new group $\tilde{G}_{l}\left(\tau_{\nu}\right)$ by the following normalization: (1) $p^{(+)}\left(1, i_{1}, \cdots, i_{\mu(l)}, 0, \cdots, 0\right)\left(\tau_{\nu}\right)=0(2) d_{l}\left(\tau_{\nu}\right)=\infty$, and $(3) p_{r}^{-}\left(\tau_{\nu}\right)=1$. We denote again by $\tilde{G}_{l}\left(\tau_{\nu}\right)$ the new group. By the similar normalizations to 
the above, we obtain new groups from $\hat{G}_{r}\left(\tau_{\nu}\right)$ and $\hat{G}_{l}^{*}\left(\tau_{\nu}\right)$, which we denote again by $\hat{G}_{r}\left(\tau_{\nu}\right)$ and $\hat{G}_{l}^{*}\left(\tau_{\nu}\right)$, respectively.

3-2. By the above normalizations, the $T_{\nu} \in$ Möb with $\tilde{G}_{l}\left(\tau_{\nu}\right)=$ $T_{\nu} \tilde{G}_{r}\left(\tau_{\nu}\right) T_{\nu}^{-1}$, has the following properties: $T_{\nu}(0)=1, T_{\nu}(1)=0$, and $T_{\nu}\left(d_{l}\left(\tau_{\nu}\right)\right)$ $=\infty$. Set $d_{l}\left(\tau_{\nu}\right)=d_{\nu}$. Then we have

$$
w=T_{\nu}(z)=\frac{d_{\nu}(z-1)}{z-d_{\nu}} \quad \text { and } \quad z=T_{\nu}^{-1}(w)=\frac{d_{\nu}(w-1)}{w-d_{\nu}} .
$$

We note that

$$
T_{\nu}^{\prime}(z)=\frac{d_{\nu}\left(1-d_{\nu}\right)}{\left(z-d_{\nu}\right)^{2}} \quad \text { and } \quad T_{\nu}^{-1^{\prime}}(w)=\frac{d_{\nu}\left(1-d_{\nu}\right)}{\left(w-d_{\nu}\right)^{2}} .
$$

Furthermore $T_{\nu}(\infty)=d_{\nu}$ and $\lim _{\nu \rightarrow \infty} d_{\nu}=1$.

We consider $p_{r, h}\left(\tau_{\nu}\right), q_{r, h}\left(\tau_{\nu}\right), h=1,2, \cdots, g_{r}$ (resp. $p_{l, h}^{*}\left(\tau_{\nu}\right)$ and $q_{l, h}^{*}\left(\tau_{\nu}\right)$, $\left.h=1,2, \cdots, g_{l}\right)$, the repelling and the attracting fixed points of $A_{r, h}\left(\tau_{\nu}, z\right)$ (resp. $A_{l, h}^{*}\left(\tau_{\nu}, z\right)$ ); $p_{r, h}\left(\tau_{\nu}\right)$ and $q_{r, h}\left(\tau_{\nu}\right)$ (resp. $p_{l, h}^{*}\left(\tau_{\nu}\right)$ and $q_{l, h}^{*}\left(\tau_{\nu}\right)$ ), the distinguished points of the first kind to the exterior to $c_{r}$ in $\omega_{r}\left(\tau_{\nu}\right)$ (resp. $c_{l}^{*}$ in $\omega_{l}\left(\tau_{\nu}\right)^{*}$ ) for $h=g_{r}+1, \cdots, g_{r}+k_{r}$ (resp. $\left.h=g_{l}+1, \cdots, g_{l}+k_{l}\right) ; p_{r, k}^{\prime}\left(\tau_{\nu}\right), \quad k=1$, $2, \cdots, n_{r}$ (resp. $p_{l, k}^{*}\left(\tau_{\nu}\right), k=1,2, \cdots, n_{l}$ ), the distinguished points of the second kind to the exterior to $c_{r}$ in $\omega_{r}\left(\tau_{\nu}\right)$ (resp. $c_{l}^{*}$ in $\left.\omega_{l}\left(\tau_{\nu}\right)^{*}\right)$. We set $p_{l, h}\left(\tau_{\nu}\right)$ $=T_{\nu}^{-1}\left(p_{l, h}^{*}\left(\tau_{\nu}\right)\right)$ for $h=1,2, \cdots, g_{l}+k_{l}$ and $p_{l, k}^{\prime}\left(\tau_{\nu}\right)=T_{\nu}^{-1}\left(p_{l, k}^{*}\left(\tau_{\nu}\right)\right)$ for $k=1$, $2, \cdots, n_{l}$. We note that $p_{l, h}\left(\tau_{\nu}\right), q_{l, h}\left(\tau_{\nu}\right)$ and $p_{l, k}^{\prime}\left(\tau_{\nu}\right)$ are all in the interior to the circle $c_{r}$.

We denote by $\hat{P}_{r, 1}\left(\tau_{\nu}\right)$ (resp. $\hat{P}_{l, 1}\left(\tau_{\nu}\right)$ ) the set of all the points $p_{r, h}\left(\tau_{\nu}\right)$, $q_{r, h}\left(\tau_{\nu}\right)$ (resp. $\left.p_{l, h}\left(\tau_{\nu}\right), q_{l, h}\left(\tau_{\nu}\right)\right)$ for $h=1,2, \cdots, g_{r}$ (resp. $h=1,2, \cdots, g_{l}$ ); by $\hat{P}_{r, 2}\left(\tau_{\nu}\right)$ (resp. $\hat{P}_{l, 2}\left(\tau_{\nu}\right)$ ) the set of all the points $p_{r, h}\left(\tau_{\nu}\right), q_{r, h}\left(\tau_{\nu}\right)$ (resp. $p_{l, h}\left(\tau_{\nu}\right)$, $\left.q_{l, h}\left(\tau_{\nu}\right)\right)$ for $h=g_{r}+1, \cdots, g_{r}+k_{r}$ (resp. $\left.g_{l}+1, \cdots, g_{l}+k_{l}\right)$; by $\hat{P}_{r, 3}\left(\tau_{\nu}\right)$ (resp. $\left.\hat{P}_{l, 3}\left(\tau_{\nu}\right)\right)$ the set of all the points $p_{r, k}^{\prime}\left(\tau_{\nu}\right)$ (resp. $\left.p_{l, k}^{\prime}\left(\tau_{\nu}\right)\right)$ for $k=1,2, \cdots$, $n_{r}$ (resp. $\left.k=1,2, \cdots, n_{l}\right)$. We set $\hat{P}_{r}\left(\tau_{\nu}\right)=\hat{P}_{r, 1}\left(\tau_{\nu}\right) \cup \hat{P}_{r, 2}\left(\tau_{\nu}\right) \cup \hat{P}_{r, 3}\left(\tau_{\nu}\right)$ and $\hat{P}_{l}\left(\tau_{\nu}\right)=\hat{P}_{l, 1}\left(\tau_{\nu}\right) \cup \hat{P}_{l, 2}\left(\tau_{\nu}\right) \cup \hat{P}_{l, 3}\left(\tau_{\nu}\right)$. For $p \in \hat{P}_{l}\left(\tau_{\nu}\right)$, we denote by $p^{*} \in \hat{P}_{l}^{*}\left(\tau_{\nu}\right)$ $=T_{\nu} \hat{P}_{l}\left(\tau_{\nu}\right) T_{\nu}^{-1}$ the image of $p$ under the mapping $T_{\nu}$.

It is not difficult to see that $\lim _{\nu \rightarrow \infty} p_{l, h}^{*}\left(\tau_{\nu}\right) \neq \infty, \lim _{\nu \rightarrow \infty} q_{l, h}^{*}\left(\tau_{\nu}\right) \neq \infty(h$ $\left.=1,2, \cdots, g_{l}+k_{l}\right), \lim _{\nu \rightarrow \infty} p_{l, k}^{\prime *}\left(\tau_{\nu}\right) \neq \infty\left(k=1,2, \cdots, n_{l}\right), \lim _{\nu \rightarrow \infty} p_{r, h}\left(\tau_{\nu}\right) \neq 1$, $\lim _{\nu \rightarrow \infty} q_{r, h}\left(\tau_{\nu}\right) \neq 1\left(h=1,2, \cdots, g_{r}+k_{r}\right)$ and $\lim _{\nu \rightarrow \infty} p_{r, k}^{\prime}\left(\tau_{\nu}\right) \neq 1\left(k=1,2, \cdots, n_{r}\right)$.

Take arbitrary $u$ elements

$$
a_{1}\left(\tau_{\nu}\right), \cdots, a_{u}\left(\tau_{\nu}\right) \in \hat{P}_{r}\left(\tau_{\nu}\right)
$$


for every $\nu$, where each $a_{i}\left(\tau_{\nu}\right)$ is one of $p_{r, h}\left(\tau_{\nu}\right), q_{r, h}\left(\tau_{\nu}\right), p_{r, k}^{\prime}\left(\tau_{\nu}\right)$ selected independently of $\nu$ (namely, the choice of $p, q, p^{\prime}$ and the suffices $h, k$ are the same for all $\nu$ ). In the same sense, we take arbitrary $v$ elements

$$
b_{1}\left(\tau_{\nu}\right), \cdots, b_{v}\left(\tau_{\nu}\right) \in \hat{P}_{l}\left(\tau_{\nu}\right) .
$$

We denote by $a_{i}^{*}\left(\tau_{\nu}\right)$ and $b_{j}^{*}\left(\tau_{\nu}\right)$ the images of $a_{i}\left(\tau_{\nu}\right)$ and $b_{j}\left(\tau_{\nu}\right)$ under the mapping $T_{\nu}$, respectively. We treat the following function:

$$
\left(\Xi \tau_{\nu}, z\right)=1 /\left(\prod_{i=1}^{u}\left(z-a_{i}\left(\tau_{\nu}\right)\right)^{m_{i}} \prod_{j=1}^{v}\left(z-b_{i}\left(\tau_{\nu}\right)\right)^{n_{j}}\right)
$$

with $m_{1}+\cdots+m_{u}+n_{1}+\cdots+n_{v}=2 q, 0 \leqq m_{i} \leqq q, 0 \leqq n_{j} \leqq q, m_{i}, n_{j}$ $\in Z$ (the set of integers) $(i=1,2, \cdots, u ; j=1,2, \cdots, v)$. Then we have the following:

(i) If $0 \leqq m_{i} \leqq q-1(i=1,2, \cdots, u), 0 \leqq n_{j} \leqq q-1(j=1,2, \cdots, v)$, then $\Xi \in M_{r}\left(\tau_{\nu}\right)$,

(ii) if two of $a_{i}\left(\tau_{\nu}\right)$ (or $b_{j}\left(\tau_{\nu}\right)$ ), say $a_{1}\left(\tau_{\nu}\right)$ and $a_{2}\left(\tau_{\nu}\right)$ (resp. $b_{1}\left(\tau_{\nu}\right)$ and $b_{2}\left(\tau_{\nu}\right)$ ), are the fixed points of $A_{r, h}\left(\tau_{\nu}, z\right), h=1,2, \cdots, g_{r}$ (resp. $A_{l, h}\left(\tau_{\nu}, z\right), h$ $\left.=1,2, \cdots, g_{l}\right)$ or the distinguished points of the first kind $p_{r, h}\left(\tau_{\nu}, z\right)$ and $q_{r, h}\left(\tau_{\nu}, z\right)$, and $m_{1}=m_{2}=q$ (resp. $\left.n_{1}=n_{2}=q\right)$, then $E \in N_{r}\left(\tau_{\nu}\right)$, and

(iii) if only one $m_{i}$, say $m_{1}$, or $n_{j}$, say $n_{1}$, is equal to $q$ and $a_{1}\left(\tau_{\nu}\right) \in$ $\hat{P}_{r, 3}\left(\tau_{\nu}\right)$ or $b_{1}\left(\tau_{\nu}\right) \in \hat{P}_{l, 3}\left(\tau_{\nu}\right)$, or if one $m_{i}$, say $m_{1}$, and one $n_{j}$, say $n_{1}$, are equal to $q$ and $a_{1}\left(\tau_{\nu}\right) \in \hat{P}_{r, 3}\left(\tau_{\nu}\right)$ and $b_{1}\left(\tau_{\nu}\right) \in \hat{P}_{l, 3}\left(\tau_{\nu}\right)$, then $\Xi \in L_{r}\left(\tau_{\nu}\right)$.

3-3. We define $\Xi^{*}(w)$ by setting $\Xi^{*}(w)=\Xi\left(T^{-1}(w)\right) T^{-1^{\prime}}(w)^{q}$. Then we have

$$
\Xi^{*}(w)=\frac{\prod_{i=1}^{u}\left(a_{i}^{*}\left(\tau_{\nu}\right)-d_{\nu}\right)^{m_{i}} \prod_{j=1}^{v}\left(b_{j}^{*}\left(\tau_{\nu}\right)-d_{\nu}\right)^{n_{j}}}{d_{\nu}^{q}\left(1-d_{\nu}\right)^{q} \prod_{i=1}^{u}\left(w-a_{i}^{*}\left(\tau_{\nu}\right)\right)^{m_{i}} \prod_{j=1}^{v}\left(w-b_{j}^{*}\left(\tau_{\nu}\right)\right)^{n_{j}}} .
$$

where $a_{i}^{*}\left(\tau_{\nu}\right)$ and $b_{j}^{*}\left(\tau_{\nu}\right)$ are the images of $a_{i}\left(\tau_{\nu}\right)$ and $b_{j}\left(\tau_{\nu}\right)$ under the mapping $T_{\nu}$. Setting

$$
\tilde{\Xi}(w)=1 /\left(\prod_{i=1}^{u}\left(w-a_{i}^{*}\left(\tau_{\nu}\right)\right)^{m_{i}} \prod_{j=1}^{v}\left(w-b_{j}^{*}\left(\tau_{\nu}\right)\right)^{n_{j}}\right)
$$

we have

$$
\Xi^{*}(w)=\tilde{\Xi}(w)\left(\prod_{i=1}^{u}\left(a_{i}^{*}\left(\tau_{\nu}\right)-d_{\nu}\right)^{m_{i}} \prod_{j=1}^{n}\left(b_{j}^{*}\left(\tau_{\nu}\right)-d_{\nu}\right)^{n_{j}} /\left(d_{\nu}^{q}\left(1-d_{\nu}\right)^{q}\right)\right) .
$$

Since $a_{i}^{*}\left(\tau_{\nu}\right)-d_{\nu}=d_{\nu}\left(d_{\nu}-1\right) /\left(a_{i}\left(\tau_{\nu}\right)-d_{\nu}\right)(i=1,2, \cdots, u)$, 


$$
\begin{gathered}
\Xi^{*}(w)=\tilde{\Xi}(w) \frac{\left(1-d_{\nu}\right)^{m_{1}+\cdots+m_{u}-q}(-1)^{m_{1}+\cdots+m_{u}}}{d_{\nu}^{q-\left(m_{1}+\cdots+m_{u}\right)}} \\
\quad \times \frac{\prod_{j=1}^{v}\left(b_{j}^{*}\left(\tau_{\nu}\right)-d_{\nu}\right)^{n_{j}}}{\prod_{i=1}^{u}\left(a_{i}\left(\tau_{\nu}\right)-d_{\nu}\right)^{m_{i}}}
\end{gathered}
$$

Since $\lim _{\nu \rightarrow \infty}\left(a_{i}\left(\tau_{\nu}\right)-d_{\nu}\right) \neq 0(i=1,2, \cdots, u)$ and $\lim _{\nu \rightarrow \infty}\left(b_{j}^{*}\left(\tau_{\nu}\right)-d_{\nu}\right) \neq \infty$ $(j=1,2, \cdots, v)$, we have the following:

LEMMA 1. (i ) If $m_{1}+\cdots+m_{u}>q$, then $\Xi^{*}\left(\tau_{0}, w\right)=\lim _{\nu \rightarrow \infty} \Xi^{*}\left(\tau_{\nu}, w\right)$ $=0$,

(ii) if $m_{1}+\cdots+m_{u}<q$, then $\Xi^{*}\left(\tau_{0}, w\right)=\infty$, and

(iii) if $m_{1}+\cdots+m_{u}=n_{1}+\cdots+n_{v}=q$, then

$$
\Xi^{*}\left(\tau_{0}, w\right)=(-1)^{q} \tilde{\Xi}\left(\tau_{0}, w\right) \frac{\prod_{j=1}^{v}\left(b_{j}^{*}\left(\tau_{0}\right)-1\right)^{n_{j}}}{\prod_{i=1}^{u}\left(a_{i}\left(\tau_{0}\right)-1\right)^{m_{i}}} .
$$

If $m_{1}+\cdots+m_{u}=n_{1}+\cdots+n_{v}=q$, we define $\Xi\left(\tau_{0}, z\right)$ as $\lim _{\nu \rightarrow \infty} \Xi\left(\tau_{\nu}, z\right)$. We have

$$
\begin{aligned}
\Xi\left(\tau_{0}, z\right) & =1 /\left((z-1)^{q} \prod_{i=1}^{u}\left(z-a_{i}\left(\tau_{0}\right)\right)^{m_{i}}\right) \\
& =1 /\left((z-1)^{q} \prod_{i=1}^{u}\left(1-a_{i}\left(\tau_{0}\right)\right)^{m_{i}}\right)+\cdots .
\end{aligned}
$$

We remember that $z=1$ is $p_{l}^{+}\left(\tau_{0}\right)$. The residue of $\Xi\left(\tau_{0}, z\right)$ at this point is equal to

$$
\operatorname{Res}_{z=1} \Xi\left(\tau_{0}, z\right)=1 /\left(\prod_{i=1}^{u}\left(1-a_{i}\left(\tau_{0}\right)\right)^{m_{i}}\right) .
$$

On the other hand,

$$
\begin{aligned}
\Xi^{*}\left(\tau_{0}, w\right) & =(-1)^{q} \frac{\prod_{j=1}^{v}\left(b_{j}^{*}\left(\tau_{0}\right)-1\right)^{n_{j}}}{(w-1)^{q} \prod_{j=1}^{v}\left(w-b_{j}^{*}\left(\tau_{0}\right)\right)^{n_{j}} \prod_{i=1}^{u}\left(a_{i}\left(\tau_{0}\right)-1\right)^{m_{i}}} \\
& =(-1)^{q} /\left((w-1)^{q} \prod_{i=1}^{u}\left(1-a_{i}\left(\tau_{0}\right)\right)^{m_{i}}\right)+\cdots .
\end{aligned}
$$

Observe that $w=1$ is $p_{l}^{-}\left(\tau_{0}\right)$. Hence we have the following:

Lemma 2.

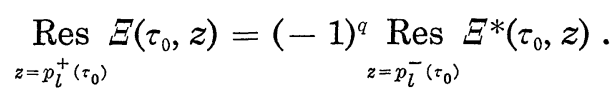

3-4. We will introduce $q$-differentials on $S(\tau)$ (see [5], p. 4) corresponding to functions $\phi_{s, h}, \theta_{s, \pi}, \psi_{j}$, and $\psi_{s, o}$ on $\sum_{t=0}^{2 g-3} \mathfrak{\mho}_{t} \hat{\Im}_{g}^{*}(\Sigma)$. 
If $\tau \in \delta^{I, J \widetilde{S}_{g}}(\Sigma)$ and $J=\left\{j_{1}, \cdots, j_{m}\right\}$, then we have

$$
S(\tau)=S_{0}(\tau)+S_{j_{1}}(\tau)+\cdots+S_{j_{m}}(\tau) .
$$

On taking $s_{i}$ with $k\left(s_{i}\right)=j_{i}$ for $i=1,2, \cdots, m$ (see Remark [5], p. 75), we have

$$
S(\tau)=\tilde{S}_{0}(\tau)+\tilde{S}_{s_{1}}(\tau)+\cdots+\tilde{S}_{s_{m}}(\tau)
$$

where $\tilde{S}_{s}(\tau)=\Omega\left(\tilde{G}_{s}(\tau)\right) / \tilde{G}_{s}(\tau)$.

We denote by $f$ an arbitrary one of $\phi_{s, h}, \theta_{s, \pi}, \psi_{j}$, and $\psi_{s, \sigma}$. Let $\pi_{s}$ be the projection of $\Omega\left(\tilde{G}_{s}(\tau)\right)$ onto $\tilde{S}_{s}(\tau)$. We define a $q$-differential $\hat{f}(\tau, \zeta) d \zeta^{q}$ on $\tilde{S}_{s}(\tau)$ by setting

$$
\hat{f}\left(\tau, \pi_{s}(z)\right) \pi_{s}^{\prime}(z)^{q}=f(\tau, z) .
$$

We have to show that the $q$-differential $\hat{f}(\tau, \zeta) d \zeta^{q}$ on $S(\tau)$ is well-defined. In fact, if $k(s)=k(t)=j$, we have to show that the $q$-differentials on $\tilde{S}_{s}(\tau)$ and $\tilde{S}_{t}(\tau)$ express the same one on $S_{j}(\tau)$.

If $s$ and $t$ are as above, then there exists $T \in$ Möb with $\tilde{G}_{t}(\tau)=$ $T \tilde{G}_{s}(\tau) T^{-1}$. Then $f(\tau, w)=f\left(\tau, T^{-}(w)\right) T^{-1^{\prime}}(w)^{q}$ for $w \in \Omega^{\prime}\left(\tilde{G}_{t}(\tau)\right)$. Set $w=$ $T(z)\left(z \in \Omega^{\prime}\left(\tilde{G}_{s}(\tau)\right)\right), \zeta=\pi_{s}(z)$ and $\omega=\pi_{t}(w)$. In this case we note that $\pi_{s}(z)=\pi_{t}(T(z))$, that is, $\zeta=\omega$, Furthermore $f(\tau, z) \pi_{s}^{\prime}(z)^{-q}=f(\tau, w) \pi_{t}^{\prime}(w)^{-q}$.

Definition. $\phi_{s, h}(\tau, z), \theta_{s, \pi}(\tau, z), \psi_{j}(\tau, z)$ or $\psi_{s, a}(\tau, z)$ is said to be a regular $q$-form if it induces a regular $q$-differential on the Riemann surface $S(\tau)$.

By modifying the proof in [2], we have the following propositions.

Proposition 2. The series (2), (5) and (7) converge absolutely for every $(\tau, z) \in \sum_{t=0}^{2 g-3} \widetilde{\mho}_{t} \hat{\Im}_{g}^{*}(\Sigma)$ and uniformly in each compact set in $\sum_{t=0}^{2 g-3} \widetilde{\mho}_{t} \hat{\Im}_{g}^{*}(\Sigma)$. For every fixed $\tau \in \hat{\subseteq}_{g}^{*}(\Sigma)$, the functions $\phi_{s, h}(\tau, z), \theta_{s, \pi}(\tau, z)$ and $\psi_{j}(\tau, z)$ are regular $q$-forms in $\Omega^{\prime}\left(\tilde{G}_{s}(\tau)\right)$. The functions $\psi_{s, \sigma}(\tau, z)$ are regular $q$-forms for fixed $\tau \in \Im_{g}^{I, J}(\Sigma)$ and $\sigma \in \mathscr{S}_{s}^{I, J}$.

Proposition 3. For every $I \subset\{1,2, \cdots, g\}$ and $J \subset\{1,2, \cdots, 2 g-3\}$, $\phi_{s, h}(\tau, z), \theta_{s, \pi}(\tau, z)$ and $\psi_{j}(\tau, z)$ are holomorphic functions of

$$
(\tau, z) \in \sum_{t=0}^{2 g-3} \widetilde{\mho}_{t} \delta^{I, v} \widetilde{S}_{g}(\Sigma)
$$

So are $\psi_{s, o}(\tau, z)$ if $\sigma \in \mathscr{S}_{s}^{I, J}$. 


\section{§4. Continuity of functions $\phi_{s, h}, \theta_{s, \pi}, \psi_{j}$ and $\psi_{s, \sigma}$}

4-1. Proposition 4. Let $I \subset\{1,2, \cdots, g\}$ with $|I|<g, J \subset\{1,2, \cdots$, $2 g-3\}$ and $k \in\{1,2, \cdots, g\} \backslash I$. Set $K=I \cup\{k\}$. Let $f$ be one of the functions $\phi_{s, h}, \theta_{s, \pi}, \psi_{j}$ and $\psi_{s, \sigma}$ with $\sigma \in \mathscr{S}_{s}^{K, J}$. If

$$
\left\{\tau_{\nu}\right\} \subset \delta^{I, J} \widetilde{S}_{g}(\Sigma), \lim _{\nu \rightarrow \infty} \tau_{\nu}=\tau_{0} \in \delta^{K, J} \widetilde{S}_{g}(\Sigma),
$$

then

$$
\lim _{\nu \rightarrow \infty} f\left(\tau_{\nu}, z\right)=f\left(\tau_{0}, z\right) \quad \text { for } z \in \Omega^{\prime}\left(\tilde{G}_{t}\left(\tau_{0}\right)\right)
$$

for $t=0,1,2, \cdots, 2 g-3$.

This is proved by modifying the proof in Bers [2].

4-2. Next we consider the deformation given in $\S 3-1$, by $\tau_{\nu} \rightarrow \tau_{0}$ with $\tau_{\nu} \in \delta^{I, J \widetilde{S}_{g}}(\Sigma), \tau_{0} \in \delta^{I, L} \widetilde{S}_{g}(\Sigma), L=J \cup\{l\}$. Notice that the number $r$ is determined by $\gamma_{l} \subset\left[\sigma_{r}(J)\right]$.

At first we remember the following: For $s$ with $k(s) \neq r, \phi_{s, h}\left(\tau_{\nu}, z\right)=0$ for $z \in \Omega^{\prime}\left(\tilde{G}_{r}\left(\tau_{\nu}\right)\right)$ or $z \in \Omega^{\prime}\left(\tilde{G}_{l}\left(\tau_{\nu}\right)\right)$, and $\phi_{s, h}\left(\tau_{0}, z\right)=0$ for $z \in \Omega^{\prime}\left(\tilde{G}_{r}\left(\tau_{0}\right)\right)$ or $z \in$ $\Omega^{\prime}\left(\tilde{G}_{l}\left(\tau_{0}\right)\right)$. With respect to $\theta_{s, \pi}$ and $\psi_{s, \sigma}$, the same holds.

For $k \notin J_{r}, \psi_{k}\left(\tau_{\nu}, z\right)=0$ for $z \in \Omega^{\prime}\left(\tilde{G}_{r}\left(\tau_{\nu}\right)\right)$ or $z \in \Omega^{\prime}\left(\tilde{G}_{l}\left(\tau_{\nu}\right)\right)$, and $\psi_{k}\left(\tau_{0}, z\right)$ $=0$ for $z \in \Omega^{\prime}\left(\tilde{G}_{r}\left(\tau_{0}\right)\right)$ or $z \in \Omega^{\prime}\left(\tilde{G}_{l}\left(\tau_{0}\right)\right)$.

Therefore we consider here $\phi_{s, h}, \theta_{s, \pi}, \psi_{s, \sigma}$ for $s$ with $k(s)=r$ and $\psi_{k}$ for $k \in J_{r}$ only. From $\S 4$ through $\S 6$, we assume that $s$ and $k$ satisfy $k(s)$ $=r$ and $k \in J_{r}$, respectively.

We recall that $G_{r}\left(\tau_{\nu}\right)=\tilde{G}_{r}\left(\tau_{\nu}\right)$ and $\tilde{G}_{l}\left(\tau_{\nu}\right)=T_{\nu} \tilde{G}_{r}\left(\tau_{\nu}\right) T_{\nu}^{-1}$. Let $F\left(\tau_{\nu}, z\right)$ be one of $\Phi_{s, h}\left(\tau_{\nu}, z\right), \Theta_{s, \pi}\left(\tau_{\nu}, z\right), \Psi_{k}\left(\tau_{\nu}, z\right)$ and $\Psi_{s, \sigma}\left(\tau_{\nu}, z\right)$. We set

$$
\begin{aligned}
& F^{*}\left(\tau_{\nu}, w\right)=F\left(\tau_{\nu}, T_{\nu}^{-1}(w)\right) T_{\nu}^{-1^{\prime}}(w)^{q} \quad \text { for } w \in \Omega^{\prime}\left(\tilde{G}_{l}\left(\tau_{\nu}\right)\right) \\
& f\left(\tau_{\nu}, z\right)=\sum_{r \in \tilde{G}_{r}\left(\tau_{\nu}\right)} F\left(\tau_{\nu}, \gamma(z)\right) \gamma^{\prime}(z)^{q} \quad \text { for } z \in \Omega^{\prime}\left(\tilde{G}_{r}\left(\tau_{\nu}\right)\right) \\
& f^{*}\left(\tau_{\nu}, w\right)=\sum_{\gamma^{*} \in \tilde{G}_{l}\left(\tau_{\nu}\right)} F^{*}\left(\tau_{\nu}, \gamma^{*}(w)\right) \gamma^{* \prime}(w)^{q} \quad \text { for } w \in \Omega^{\prime}\left(\tilde{G}_{l}\left(\tau_{\nu}\right)\right) .
\end{aligned}
$$

By means of $F\left(\tau_{0}, z\right)$ with the similar meaning, we define $f\left(\tau_{0}, z\right), z \in \Omega^{\prime}\left(\tilde{G}_{r}\left(\tau_{0}\right)\right)$ and $f^{*}\left(\tau_{0}, w\right), w \in \Omega^{\prime}\left(\tilde{G}_{l}\left(\tau_{0}\right)\right)$ by setting

$$
f\left(\tau_{0}, z\right)=\sum_{\tilde{G}_{r}\left(\tau_{0}\right)} F\left(\tau_{0}, \gamma(z)\right) \gamma^{\prime}(z)^{q} \quad \text { for } z \in \Omega^{\prime}\left(\tilde{G}_{r}\left(\tau_{0}\right)\right)
$$

and

$$
f^{*}\left(\tau_{0},{ }^{p} w\right)=\sum_{\gamma^{*} \in \tilde{G}_{l}\left(\tau_{0}\right)} F^{*}\left(\tau_{0}, \gamma^{*}(w)\right) \gamma^{* \prime}(w)^{q} \quad \text { for } w \in \Omega^{\prime}\left(\tilde{G}_{l}\left(\tau_{0}\right)\right)
$$


where $F^{*}\left(\tau_{0}, w\right)=\lim _{\nu \rightarrow \infty} F^{*}\left(\tau_{\nu}, w\right)$.

4-3. Let $I \subset\{1,2, \cdots, g\}$ and $J \subset\{1,2, \cdots, 2 g-3\}, \quad|J|<2 g-3$. Let $l \in\{1,2, \cdots, 2 g-3\} \mid J$. Set $L=J \cup\{l\}$. We denote by $W_{r}(J)$ (resp. $\left.W_{r}(L)\right)$ the set $\{s \mid k(s)=r\}$ for $J$ (resp. $\left.L\right)$. In general, $W_{r}(J)$ may not coincide with $W_{r}(L)$.

Proposition 5. Let $I, J, l, L$ be as in the above. Suppose

$$
\left\{\tau_{\nu}\right\} \subset \delta^{I, J} \Im_{g}(\Sigma), \lim _{\nu \rightarrow \infty} \tau_{\nu}=\tau_{0} \in \delta^{I, L} \widetilde{S}_{g}(\Sigma) .
$$

If $F$ is one of the functions $\Theta_{s, \pi}\left(\tau_{\nu}, z\right) \in M_{s}\left(\tau_{\nu}\right)$ such that $\Theta_{s, \pi}\left(\tau_{0}, z\right) \in M_{s}\left(\tau_{0}\right)$ with $s \in W_{r}(L)$, then

$$
\lim _{\nu \rightarrow \infty} f\left(\tau_{\nu}, z\right)=f\left(\tau_{0}, z\right) \quad \text { for } z \in \Omega^{\prime}\left(\tilde{G}_{r}\left(\tau_{0}\right)\right) .
$$

Here $f$ are defined from $F$ in $\S 4-2$.

Proof. It suffices to consider the following functions:

$$
F\left(\tau_{\nu}, z\right)=1 /\left(\prod_{i=1}^{u}\left(z-a_{i}\left(\tau_{\nu}\right)\right)^{m_{i}} \prod_{j=1}^{v}\left(z-b_{j}\left(\tau_{\nu}\right)\right)^{n_{j}}\right) \quad \tau_{\nu} \in \delta^{I, J \Im_{g}}(\Sigma)
$$

and

$$
F\left(\tau_{0}, z\right)=\lim _{\nu \rightarrow \infty} F\left(\tau_{\nu}, z\right)
$$

with $a_{i}\left(\tau_{\nu}\right) \in \hat{P}_{r}\left(\tau_{\nu}\right)(i=1,2, \cdots, u), b_{j}\left(\tau_{\nu}\right) \in \hat{P}_{l}\left(\tau_{\nu}\right)(j=1,2, \cdots, v), m_{1}+\cdots$ $+m_{u}>q>n_{1}+\cdots+n_{v}, 1 \leqq m_{i} \leqq q-1,1 \leqq n_{j} \leqq q-1, m_{i}, n_{j} \in Z$, where $a_{i}\left(\tau_{\nu}\right)$ and $b_{j}\left(\tau_{\nu}\right)$ are selected as in $\S 3-2$, pp. 87-88.

We will prove the proposition by modifying the method in [2]. Let $\hat{G}_{r}\left(\tau_{\nu}\right)=\left\langle A_{r, 1}\left(\tau_{\nu}, z\right), \cdots, A_{r, g_{r}}\left(\tau_{\nu}, z\right)\right\rangle$ be the subgroup of $\tilde{G}_{r}\left(\tau_{\nu}\right)$ defined in $\S 3-1$. Let

$$
\tilde{G}_{r}\left(\tau_{\nu}\right)=\hat{G}_{r}\left(\tau_{\nu}\right) \gamma_{0}\left(\tau_{\nu}\right)+\hat{G}_{r}\left(\tau_{\nu}\right) \gamma_{1}\left(\tau_{\nu}\right)+\hat{G}_{r}\left(\tau_{\nu}\right) \gamma_{2}\left(\tau_{\nu}\right)+\cdots,
$$

where $\gamma_{0}\left(\tau_{\nu}\right)=\mathrm{id}, \gamma_{1}\left(\tau_{\nu}\right), \gamma_{2}\left(\tau_{\nu}\right), \ldots$ is a complete list of the right coset representatives of $\tilde{G}_{r}\left(\tau_{\nu}\right)$ modulo $\hat{G}_{r}\left(\tau_{\nu}\right)$. Set

$$
\hat{F}\left(\tau_{\nu}, z\right)=\sum_{r \in \tilde{G}_{r}\left(\tau_{\nu}\right)} F\left(\tau_{\nu}, \gamma(z)\right) \gamma^{\prime}(z)^{q} .
$$

By the same way as in the proofs of Propositions 2 and 3, we see that this series converges absolutely, and depends holomorphically on $(\tau, z)$ $\in \mathfrak{F}_{r} \mathbb{S}_{g}^{I, L}(\Sigma)$. We have 


$$
\hat{F}\left(\tau_{0}, z\right)=\sum_{\gamma \in \tilde{G}_{r}\left(\tau_{0}\right)} F\left(\tau_{0}, \gamma(z)\right) \gamma^{\prime}(z)^{q}=f\left(\tau_{0}, z\right)
$$

and

$$
\begin{aligned}
f\left(\tau_{\nu}, z\right) & =\sum_{r \in \tilde{\sigma}_{r}\left(\tau_{\nu}\right)} F\left(\tau_{\nu}, \gamma(z)\right) \gamma^{\prime}(z)^{q} \\
& =\sum_{n=0}^{\infty} \hat{F}\left(\tau_{\nu}, \gamma_{n}(z)\right) \gamma_{n}^{\prime}(z)^{q} \\
& =\hat{F}\left(\tau_{\nu}, z\right)+\sum_{n=1}^{\infty} \hat{F}\left(\tau_{\nu}, \gamma_{n}(z)\right) \gamma_{n}^{\prime}(z)^{q},
\end{aligned}
$$

where we write $\gamma_{n}$ for $\gamma_{n}\left(\tau_{\nu}\right)$ for simplicity. Set

$$
R_{\nu}(z)=\sum_{n=1}^{\infty} \hat{F}\left(\tau_{\nu}, \gamma_{n}(z)\right) \gamma_{n}^{\prime}(z)^{q} .
$$

Since $\lim _{\nu \rightarrow \infty} \hat{F}\left(\tau_{\nu}, z\right)=F\left(\tau_{0}, z\right)$ by Proposition 3 , it suffices to show that

$$
\lim _{\nu \rightarrow \infty} R_{\nu}(z)=0 \quad \text { for } z \in \Omega^{\prime}\left(\tilde{G}_{r}\left(\tau_{0}\right)\right)
$$

For every large $\nu$, we can choose defining curves of $\tilde{G}_{r}\left(\tau_{\nu}\right)$ (the boundary curves of a standard fundamental domain $\omega_{r}\left(\tau_{\nu}\right)$ for $\tilde{G}_{r}\left(\tau_{\nu}\right)$ ) as follows: (1) $g_{r}$ fixed curves $C_{r, h}\left(h=1,2, \cdots, g_{r}\right)$ are independent of $\nu$. (2) Each $C_{r, h}$ surrounds the point $p_{r, h}\left(\tau_{0}\right)$. (3) $g_{r}$ curves $C_{r, h}^{\prime}\left(\tau_{\nu}\right)=A_{r, h}\left(\tau_{\nu}, C_{r, h}\right)$. (4) Each curve $C_{l, h}\left(\tau_{\nu}\right)$ surrounds the point $p_{l, h}\left(\tau_{\nu}\right), h=1,2, \cdots, g_{l}$. (5) $C_{l, h}^{\prime}\left(\tau_{\nu}\right)=$ $A_{l, h}\left(\tau_{\nu}, C_{l, h}\left(\tau_{\nu}\right)\right)$. (6) All $C_{l, h}\left(\tau_{\nu}\right)$ and $C_{l, h}^{\prime}\left(\tau_{\nu}\right)$ are contained in small disks $K\left(\tau_{\nu}\right)$ with (diameters of $\left.K\left(\tau_{\nu}\right)\right)=o(1)(\nu \rightarrow \infty)$. Let the $2 g_{r}$-ply connected region bounded by the $2 g_{r}$ curves $C_{r, h}$ and $C_{r, h}^{\prime}\left(\tau_{\nu}\right)\left(h=1,2, \cdots, g_{r}\right)$ be denoted by $\hat{\omega}_{r}\left(\tau_{\nu}\right)$. Then $\hat{\omega}_{r}\left(\tau_{\nu}\right)$ is a standard fundamental domain for $\hat{G}_{1}\left(\tau_{\nu}\right)$. We remark that $\lim _{\nu \rightarrow \infty} C_{r, h}^{\prime}\left(\tau_{\nu}\right)=C_{r, h}^{\prime}\left(\tau_{0}\right)$ and $C_{r, h}$ are $2 g_{r}$ boundary curves of a fundamental domain $\omega_{r}\left(\tau_{0}\right)$ for $\tilde{G}_{r}\left(\tau_{0}\right)$.

By using Proposition 1, we have

$$
\iint_{\hat{\omega}_{r}\left(\tau_{\nu}\right)}\left|\hat{F}\left(\tau_{\nu}, z\right)\right| \lambda\left(\tau_{\nu}, z\right)^{2-q} d x d y=O(1), \quad \nu \longrightarrow \infty,
$$

where $\lambda\left(\tau_{\nu}, z\right)|d z|$ is the Poincaré metric on $\Omega^{\prime}\left(\hat{G}_{r}\left(\tau_{\nu}\right)\right)$. From this we obtain the following: For every large $\nu$ and for a properly chosen fixed circle $C$ surrounding the disk $K\left(\tau_{\nu}\right)$,

$$
\max _{z \in C}\left|\hat{F}\left(\tau_{\nu}, z\right)\right|=O(1), \quad \nu \longrightarrow \infty \text { (uniform in } \tau \text { ). }
$$

$\hat{F}\left(\tau_{\nu}, z\right) \prod_{j=1}^{v}\left(z-b_{j}\left(\tau_{\nu}\right)\right)^{n_{j}}$, as a function of $z$, is holomorphic in the interior to $C$ (we denote it by [C]). Hence for every large $\nu$ and $z$ in [C], 
there exists a constant $M$ independent of $\nu$ such that

$$
\left|\hat{F}\left(\tau_{\nu}, z\right) \prod_{j=1}^{v}\left(z-b_{j}\left(\tau_{\nu}\right)\right)^{n_{j}}\right| \leqq M .
$$

Let $p\left(\tau_{\nu}\right) \in \hat{P}_{l}\left(\tau_{\nu}\right)$. We denote by $\Lambda_{p\left(\tau_{\nu}\right)}\left(\tau_{\nu}, z\right)|d z|$ the Poincaré metric on $\hat{\boldsymbol{C}} \backslash\left\{p_{r, 1}\left(\tau_{\nu}\right), q_{r, 1}\left(\tau_{\nu}\right), p\left(\tau_{\nu}\right)\right\}$ if $g_{r}+k_{r} \geqq 1$ (or $\hat{C} \backslash\left\{p_{r, 1}^{\prime}\left(\tau_{\nu}\right), p_{r, 2}^{\prime}\left(\tau_{\nu}\right), p\left(\tau_{\nu}\right)\right\}$ if $g_{r}+k_{r}$ $=0)$. Then we have the inequality

$$
\lambda\left(\tau_{\nu}, z\right) \geqq \Lambda_{p\left(\tau_{\nu}\right)}\left(\tau_{\nu}, z\right) .
$$

Furthermore in a neighborhood of $p\left(\tau_{\nu}\right)$,

$$
\Lambda_{p\left(\tau_{\nu}\right)}\left(\tau_{\nu}, z\right) \sim\left|z-p\left(\tau_{\nu}\right)\right|^{-1}\left(-\log \left|z-p\left(\tau_{\nu}\right)\right|\right)^{-1}
$$

(see [2], p. 343).

Hence in the interior to $C_{l, h}\left(\tau_{\nu}\right), h=1,2, \cdots, g_{l}$, (we denote them by $\left.\left[C_{l, h}\left(\tau_{\nu}\right)\right]\right)$, there exists a constant $M_{1}$ independent of $\nu$ such that

$$
\left|\hat{F}\left(\tau_{\nu}, z\right)\right| \lambda\left(\tau_{\nu}, z\right)^{2-q} \leqq M_{1}\left|z-p_{l, h}\left(\tau_{\nu}\right)\right|^{-3 / 2} .
$$

Similarly, in the interior to $C_{l, h}^{\prime}\left(\tau_{\nu}\right), h=1,2, \cdots, g_{l}$, (we denote them by $\left.\left[C_{l, h}^{\prime}\left(\tau_{\nu}\right)\right]\right)$, there exists a constant $M_{1}^{\prime}$ independent of $\nu$ such that

$$
\left|\hat{F}\left(\tau_{\nu}, z\right)\right| \lambda\left(\tau_{\nu}, z\right)^{2-q} \leqq M_{1}^{\prime}\left|z-q_{l, h}\left(\tau_{\nu}\right)\right|^{-3 / 2} .
$$

We can choose the representatives $\gamma_{1}, \gamma_{2}, \cdots$ of (15) as follows:

$$
\gamma_{n}\left(\omega_{r}\left(\tau_{\nu}\right)\right) \subset \bigcup_{h=1}^{g_{l}}\left(\left[C_{l, h}\left(\tau_{\nu}\right)\right] \cup\left[C_{l, h}^{\prime}\left(\tau_{\nu}\right)\right]\right) .
$$

Since $\gamma_{n}\left(\omega_{r}\left(\tau_{\nu}\right)\right) \cap \gamma_{m}\left(\omega_{r}\left(\tau_{\nu}\right)\right)=\phi$ for $m \neq n$,

$$
\gamma_{1}\left(\omega_{r}\left(\tau_{\nu}\right)\right) \cup \gamma_{2}\left(\omega_{r}\left(\tau_{\nu}\right)\right) \cup \cdots \subset \hat{\omega}_{r}\left(\tau_{\nu}\right) \backslash \omega_{r}\left(\tau_{\nu}\right) .
$$

Thus

$$
\begin{aligned}
& \iint_{\omega_{r}\left(\tau_{\nu}\right)}\left|R_{\nu}(z)\right| \lambda\left(\tau_{\nu}, z\right)^{2-q} d x d y \\
& \quad \leqq \sum_{n=1}^{\infty} \iint_{\omega_{r}\left(\tau_{\nu}\right)}\left|\hat{F}\left(\tau_{\nu}, \gamma_{n}(z)\right)\right|\left|\gamma_{n}^{\prime}(z)\right|^{q} \lambda\left(\tau_{\nu}, z\right)^{2-q} d x d y \\
& \quad=\sum_{n=1}^{\infty} \iint_{\gamma_{n}\left(\omega_{r}\left(\tau_{\nu}\right)\right)}\left|\hat{F}\left(\tau_{\nu}, z\right)\right| \lambda\left(\tau_{\nu}, z\right)^{2-q} d x d y \\
& \quad \leqq \iint_{\omega_{r}\left(\tau_{\nu}\right)\left(\omega_{r}\left(\tau_{\nu}\right)\right.}\left|\hat{F}\left(\tau_{\nu}, z\right)\right| \lambda\left(\tau_{\nu}, z\right)^{2-q} d x d y \\
& \quad \leqq \sum_{h=1}^{g_{l}}\left\{\iint_{\left[C_{l, h}\left(\tau_{\nu}\right)\right]} M_{1}\left|z-p_{l, h}\left(\tau_{\nu}\right)\right|^{-3 / 2} d x d y\right. \\
&\left.\quad+\iint_{\left[C_{l, h}^{\prime}\left(\tau_{\nu}\right)\right]} M_{1}^{\prime}\left|z-q_{l, h}\left(\tau_{\nu}\right)\right|^{-3 / 2} d x d y\right\}
\end{aligned}
$$


Since the diameters of $C_{l, h}\left(\tau_{\nu}\right)$ and $C_{l, h}^{\prime}\left(\tau_{\nu}\right)$ tend to 0 as $\nu \rightarrow \infty$, we have

$$
\lim _{\nu \rightarrow \infty} \iint_{\omega_{r}\left(\tau_{\nu}\right)}\left|R_{\nu}(z)\right| \lambda\left(\tau_{\nu}, z\right)^{2-q} d x d y=0
$$

Hence we obtain (13). Our proof is now complete.

4-4. Proposition 6. Under the same assumption as in Proposition 5,

$$
\lim _{\nu \rightarrow \infty} f^{*}\left(\tau_{\nu}, w\right)=f^{*}\left(\tau_{0}, w\right) \quad \text { for } w \in \Omega^{\prime}\left(\tilde{G}_{l}\left(\tau_{0}\right)\right) .
$$

Here $f^{*}$ are defined from $F$ in $\S 4-2$.

Proof. By Lemma 1, $F^{*}\left(\tau_{0}, w\right)=\lim _{\nu \rightarrow \infty} F^{*}\left(\tau_{\nu}, w\right)=0$. Since

$$
f^{*}\left(\tau_{0}, w\right)=\sum_{r^{*} \in \tilde{\sigma}_{l}\left(\tau_{0}\right)} F^{*}\left(\tau_{0}, \gamma^{*}(w)\right) \gamma^{* \prime}(w)^{q},
$$

we have $f^{*}\left(\tau_{0}, w\right)=0$. Hence it suffices to show that

$$
\lim _{\nu \rightarrow \infty} f^{*}\left(\tau_{\nu}, w\right)=0 \quad \text { for } w \in \Omega^{\prime}\left(\tilde{G}_{l}\left(\tau_{\nu}\right)\right) .
$$

We decompose $\tilde{G}_{l}\left(\tau_{\nu}\right)$ into the following two sets:

$$
\begin{aligned}
G_{1 l}^{*}\left(\tau_{\nu}\right)=\left\{\gamma^{*} \in \tilde{G}_{l}\left(\tau_{\nu}\right) \mid \gamma^{*}(w) \in\left[C_{r, 1}^{*}\left(\tau_{\nu}\right)\right] \cup\left[C_{r, 1}^{* \prime}\left(\tau_{\nu}\right)\right]\right. \\
\left.\cup \cdots \cup\left[C_{r, g_{r}}^{*}\left(\tau_{\nu}\right)\right] \cup\left[C_{r, g_{r}}^{* \prime}\left(\tau_{\nu}\right)\right] \text { for some } w \in \omega_{l}\left(\tau_{\nu}\right)^{*}\right\},
\end{aligned}
$$

where $C_{r, h}^{*}\left(\tau_{\nu}\right)=T_{\nu}\left(C_{r, h}\right)$ and $C_{r, h}^{* \prime}\left(\tau_{\nu}\right)=T_{\nu}\left(C_{r, h}^{\prime}\left(\tau_{\nu}\right)\right)\left(h=1,2, \cdots, g_{r}\right)$, and

$$
G_{2 l}^{*}\left(\tau_{\nu}\right)=\tilde{G}_{l}\left(\tau_{\nu}\right) \backslash G_{1 l}^{*}\left(\tau_{\nu}\right) \text {. }
$$

We note that an element $\gamma^{*}$ of $G_{2 l}^{*}\left(\tau_{\nu}\right)$ is the identity or satisfies

$$
\gamma^{*}(w) \in\left[C_{l, 1}^{*}\left(\tau_{\nu}\right)\right] \cup\left[C_{l, 1}^{* \prime}\left(\tau_{\nu}\right)\right] \cup \cdots \cup\left[C_{l, g_{l}}^{* \prime}\left(\tau_{\nu}\right)\right]
$$

for $w \in \omega_{l}\left(\tau_{\nu}\right)^{*}$, where $C_{l, h}^{*}\left(\tau_{\nu}\right)=T_{\nu}\left(C_{l, h}\left(\tau_{\nu}\right)\right)$ and $C_{l, h}^{* \prime}\left(\tau_{\nu}\right)=T_{\nu}\left(C_{l, h}^{\prime}\left(\tau_{\nu}\right)\right)$. We write

$$
\begin{aligned}
f^{*}\left(\tau_{\nu}, w\right)= & \sum_{r^{*} \in G_{12}^{*}\left(\tau_{\nu}\right)} F^{*}\left(\tau_{\nu}, \gamma^{*}(w)\right) \gamma^{* \prime}(w)^{q} \\
& +\sum_{\gamma^{*} \in G_{22}^{*}\left(\tau_{\nu}\right)} F^{*}\left(\tau_{\nu}, \gamma^{*}(w)\right) \gamma^{* \prime}(w)^{q} \\
= & f_{1}^{*}\left(\tau_{\nu}, w\right)+f_{2}^{*}\left(\tau_{\nu}, w\right) .
\end{aligned}
$$

We have Proposition 6 if the followings are proved:

$$
\lim _{\nu \rightarrow \infty} f_{1}^{*}\left(\tau_{\nu}, w\right)=0
$$

and 


$$
\lim _{\nu \rightarrow \infty} f_{2}^{*}\left(\tau_{\nu}, w\right)=0 \text {, }
$$

which are Propositions 7 and 8, respectively, proved in the next section.

\section{§5. Proposition 7 and Proposition 8}

5-1. Proposition 7. Under the same assumption as in Proposition 5,

$$
\lim _{\nu \rightarrow \infty} f_{1}^{*}\left(\tau_{\nu}, w\right)=0 \quad \text { for } w \in \Omega^{\prime}\left(\tilde{G}_{l}\left(\tau_{0}\right)\right) .
$$

Here $f_{1}^{*}$ are defined in the proof of Proposition 6.

Proposition 8. Under the same assumption as in Proposition 5,

$$
\lim _{\nu \rightarrow \infty} f_{2}^{*}\left(\tau_{\nu}, w\right)=0 \quad \text { for } w \in \Omega^{\prime}\left(\tilde{G}_{l}\left(\tau_{0}\right)\right) .
$$

Here $f_{2}^{*}$ are defined in the proof of Proposition 5.

The proofs of Propositions 7 and 8 will be presented in a series of the following lemmas. We need to introduce some notations in order to state the lemmas.

We set $G_{1 l}\left(\tau_{\nu}\right)=T_{\nu}^{-1} G_{1 l}^{*}\left(\tau_{\nu}\right) T_{\nu}, G_{2 l}\left(\tau_{\nu}\right)=T_{\nu}^{-1} G_{2 l}^{*}\left(\tau_{\nu}\right) T_{\nu}$,

$$
\begin{aligned}
& G_{r, h}\left(\tau_{\nu}\right)=\left\{\gamma \in \tilde{G}_{r}\left(\tau_{\nu}\right) \mid \gamma(z) \in\left[C_{r, h}\right] \text { for some } z \in \omega_{r}\left(\tau_{\nu}\right)\right\}, \\
& \left(h=1,2, \cdots, g_{r}\right) \\
& G_{r, h}^{\prime}\left(\tau_{\nu}\right)=\left\{\gamma \in \tilde{G}_{r}\left(\tau_{\nu}\right) \mid \gamma(z) \in\left[C_{r, h}^{\prime}\left(\tau_{\nu}\right)\right] \text { for some } z \in \omega_{r}\left(\tau_{\nu}\right)\right\} \text {, } \\
& \left(h=1,2, \cdots, g_{r}\right) \\
& \bar{G}_{r, h}\left(\tau_{\nu}\right)=\tilde{G}_{r}\left(\tau_{\nu}\right) \backslash\left(G_{r, h}\left(\tau_{\nu}\right) \cup G_{r, h}^{\prime}\left(\tau_{\nu}\right)\right), \quad\left(h=1,2, \cdots, g_{r}\right), \\
& G_{111}\left(\tau_{\nu}\right)=\left\{\gamma\left(\tau_{\nu}, z\right)=A_{r, h}\left(W\left(A_{r, k}\left(\tau_{\nu}, z\right)\right)\right) \in G_{1 l}\left(\tau_{\nu}\right) \mid W \in \tilde{G}_{r}\left(\tau_{\nu}\right)\right. \text {, } \\
& \left.h, k=1,2, \cdots, g_{r}\right\} \text {. } \\
& G_{112}\left(\tau_{\nu}\right)=\left\{\gamma\left(\tau_{\nu}, z\right)=W_{1}\left(W_{2}\left(\tau_{\nu}, z\right)\right) \in G_{1 l}\left(\tau_{\nu}\right) \mid W_{1}\left(\tau_{\nu}, z\right)\right. \\
& =A_{r, h}\left(V\left(A_{r, k}\left(\tau_{\nu}, z\right)\right) \text { with } h, k=1,2, \cdots, g_{r}\right. \text {, } \\
& \left.V \in \tilde{G}_{r}\left(\tau_{\nu}\right) \text {, and } W_{2} \in G_{2 l}\left(\tau_{\nu}\right)\right\} \text {. }
\end{aligned}
$$

5-2. Lemma 3. (1) For every large $\nu$, there exists a constant $M_{2}$ independent of $\nu$ such that

$$
\sum_{r \in G_{111}\left(\tau_{\nu}\right)}\left|\gamma^{\prime}\left(T_{\nu}^{-1}(w)\right)\right|^{q} \leqq M_{2} \quad \text { for } w \in \Omega^{\prime}\left(\tilde{G}_{l}\left(\tau_{0}\right)\right) .
$$

(2) For every large $\nu$, there exists a constant $M_{3}$ independent of $\nu$ such that

$$
\sum_{r \in G_{112}\left(\tau_{\nu}\right)}\left|\gamma^{\prime}\left(T_{\nu}^{-1}(w)\right)\right|^{q} \leqq M_{3} \quad \text { for } w \in \Omega^{\prime}\left(\tilde{G}_{l}\left(\tau_{0}\right)\right)
$$


(3) For every large $\nu$, there exists a constant $\mid M_{4}$ independent of $\nu$ such that

$$
\sum_{r^{*} \in G_{2 l}^{*}\left(\tau_{\nu}\right)}\left|\gamma^{* \prime}(w)\right|^{q} \leqq M_{4} \quad \text { for } w \in \Omega^{\prime}\left(\tilde{G}_{l}\left(\tau_{0}\right)\right) .
$$

Proof. (1) We set $\gamma \in G_{111}\left(\tau_{\nu}\right)$ as

$$
\gamma=\left(\begin{array}{ll}
a_{\gamma}\left(\tau_{\nu}\right) & b_{\gamma}\left(\tau_{\nu}\right) \\
c_{\gamma}\left(\tau_{\nu}\right) & d_{\gamma}\left(\tau_{\nu}\right)
\end{array}\right), \quad a_{r}\left(\tau_{\nu}\right) d_{r}\left(\tau_{\nu}\right)-b_{\gamma}\left(\tau_{\nu}\right) c_{\gamma}\left(\tau_{\nu}\right)=1 .
$$

We have

$$
\sum_{\gamma \in G_{111}\left(\tau_{\nu}\right)}\left|\gamma^{\prime}\left(T_{\nu}^{-1}(w)\right)\right|^{q}=\sum \frac{1}{\left|c_{\gamma}\left(\tau_{\nu}\right)\right|^{2 q}} \frac{1}{\left|T_{\nu}^{-1}(w)+\left(d_{\gamma}\left(\tau_{\nu}\right) / c_{\gamma}\left(\tau_{\nu}\right)\right)\right|^{2 q}} .
$$

Since

$$
-d_{r}\left(\tau_{\nu}\right) / c_{r}\left(\tau_{\nu}\right) \in \bigcup_{h=1}^{g_{r}}\left(\left[C_{r, h}\right] \cup\left[C_{r, h}^{\prime}\left(\tau_{\nu}\right)\right]\right)
$$

and $T_{\nu}^{-1}(w)$ is sufficiently close to 1 for every large $\nu$, there exists a positive constant $\delta_{1}$ independent of $\nu$ such that

$$
\left|T_{\nu}^{-1}(w)+\left(d_{\gamma}\left(\tau_{\nu}\right) / c_{\gamma}\left(\tau_{\nu}\right)\right)\right| \geqq \delta_{1} \quad \text { for all } \gamma \in G_{1 l 1}\left(\tau_{\nu}\right) .
$$

By modifying the method of Ford ([3], pp. 104-105), there exists a constant $M$ independent of $\nu$ such that

$$
\sum_{G_{111}\left(\tau_{\nu}\right)} 1 /\left|c_{\gamma}\left(\tau_{\nu}\right)\right|^{2 q} \leqq \sum_{\sigma_{r}\left(\tau_{\nu}\right)} 1 /\left|c_{\gamma}\left(\tau_{\nu}\right)\right|^{2 q} \leqq M
$$

for every large $\nu$. Therefore, for every large $\nu$,

$$
\sum_{G_{111(\tau \nu)}}\left|\gamma^{\prime}\left(T_{\nu}^{-1}(w)\right)\right|^{q} \leqq M / \delta_{1}^{2 q} \quad \text { for } w \in \Omega^{\prime}\left(\tilde{G}_{l}\left(\tau_{0}\right)\right)
$$

(2) Since $\gamma\left(T_{\nu}^{-1}(w)\right)=W_{1}\left(W_{2}\left(T_{\nu}^{-1}(w)\right)\right)$,

$$
\gamma^{\prime}\left(T_{\nu}^{-1}(w)\right)=W_{1}^{\prime}\left(W_{2}\left(T_{\nu}^{-1}(w)\right)\right) W_{2}^{\prime}\left(T_{\nu}^{-1}(w)\right) .
$$

Therefore

$$
\begin{aligned}
& \sum \mid W_{1}^{\prime}\left(\left.W_{2}\left(T_{\nu}^{-1}(w)\right) W_{2}^{\prime}\left(T_{\nu}^{-1}(w)\right)\right|^{q}\right. \\
& \quad \leqq \sum_{W_{2}}\left(\left|W_{2}^{\prime}\left(T_{\nu}^{-1}(w)\right)\right|^{q} \sum_{W_{1}}\left|W_{1}\left(W_{2}\left(T_{\nu}^{-1}(w)\right)\right)\right|^{q}\right) .
\end{aligned}
$$

Set

$$
W_{1}=\left(\begin{array}{ll}
a\left(\tau_{\nu}\right) & b\left(\tau_{\nu}\right) \\
c\left(\tau_{\nu}\right) & d\left(\tau_{\nu}\right)
\end{array}\right), \quad a\left(\tau_{\nu}\right) d\left(\tau_{\nu}\right)-b\left(\tau_{\nu}\right) c\left(\tau_{\nu}\right)=1
$$


Then we have

$$
\left|W_{1}^{\prime}\left(W_{2}\left(T_{\nu}^{-1}(w)\right)\right)\right|=\frac{1}{\left|c\left(\tau_{\nu}\right)\right|^{2}} \frac{1}{\left|W_{2}\left(T_{\nu}^{-1}(w)\right)+\left(d\left(\tau_{\nu}\right) / c\left(\tau_{\nu}\right)\right)\right|^{2}} .
$$

Since

$$
W_{2}\left(T_{\nu}^{-1}(w)\right) \in \bigcup_{h=1}^{g_{l}}\left(\left[C_{l, h}\left(\tau_{\nu}\right)\right] \cup\left[C_{l, h}^{\prime}\left(\tau_{\nu}\right)\right]\right) \quad \text { for all } W_{2} \in G_{2 l}\left(\tau_{\nu}\right)
$$

and

$$
-d\left(\tau_{\nu}\right) / c\left(\tau_{\nu}\right)=W_{1}^{-1}(\infty) \in\left[C_{r, h}\right],
$$

there exists a positive constant $\delta_{2}$ independent of $\nu$ such that

$$
\left|W_{2}\left(T_{\nu}^{-1}(w)\right)+\left(d\left(\tau_{\nu}\right) / c\left(\tau_{\nu}\right)\right)\right| \geqq \delta_{2} \quad \text { for all } W_{2} \in G_{2 l}\left(\tau_{\nu}\right) .
$$

By modifying the method of Ford ([3], pp. 104-105), there exists a constant $M$ independent of $\nu$ such that

$$
\sum_{G_{111}\left(\tau_{\nu}\right)} 1 /\left|c\left(\tau_{\nu}\right)\right|^{2 q} \leqq \sum_{\tilde{\sigma}_{r}\left(\tau_{\nu}\right)} 1 /\left|c\left(\tau_{\nu}\right)\right|^{2 q} \leqq M
$$

Therefore

$$
\sum_{W_{1} \in G_{111}\left(\tau_{\nu}\right)}\left|W_{1}^{\prime}\left(W_{2}\left(T_{\nu}^{-1}(w)\right)\right)\right|^{q} \leqq M / \delta_{2}^{2 q}
$$

for all $W_{2} \in G_{2 l}\left(\tau_{\nu}\right)$ and for every large $\nu$. Since $T_{\nu} W_{2} T_{\nu}^{-1} \in G_{2 l}^{*}\left(\tau_{\nu}\right)$ (we denote it by $W_{2}^{*}$ ),

$$
W_{2}^{\prime}\left(T_{\nu}^{-1}(w)\right)=W_{2}^{* \prime}\left(\tau_{\nu}\right) \frac{\left(w-d_{\nu}\right)^{2}}{\left(W_{2}^{*}(w)-d_{\nu}\right)^{2}} .
$$

For every large $\nu$, there exists a positiv€ constant $\delta_{3}$ independent of $\nu$ such that

$$
\left|W_{2}^{*}(w)-d_{\nu}\right| \geqq \delta_{3}
$$

Set

$$
W_{2}^{*}=\left(\begin{array}{ll}
a^{*}\left(\tau_{\nu}\right) & b^{*}\left(\tau_{\nu}\right) \\
c^{*}\left(\tau_{\nu}\right) & d^{*}\left(\tau_{\nu}\right)
\end{array}\right), \quad a^{*}\left(\tau_{\nu}\right) d^{*}\left(\tau_{\nu}\right)-b^{*}\left(\tau_{\nu}\right) c^{*}\left(\tau_{\nu}\right)=1
$$

Then

$$
\left|W_{2}^{* \prime}(w)\right|=\frac{1}{\left|c^{*}\left(\tau_{\nu}\right)\right|^{2}} \frac{1}{\left|w+\left(d^{*}\left(\tau_{\nu}\right) / c^{*}\left(\tau_{\nu}\right)\right)\right|^{2}} .
$$


For every large $\nu$, there exists a constant $M$ independent of $\nu$ such that

$$
\frac{\left|w-d_{\nu}\right|}{\left|w+\left(d^{*}\left(\tau_{\nu}\right) / c^{*}\left(\tau_{\nu}\right)\right)\right|} \leqq M \quad \text { for all } W_{2}^{*} \in G_{2 l}^{*}\left(\tau_{\nu}\right)
$$

Furthermore by modifying the method of Ford ([3] pp. 104-105), there exists a constant $M^{\prime}$ independent of $\nu$ such that

$$
\sum_{G_{2 l}^{*}\left(\tau_{\nu}\right)}\left|1 / c^{*}\left(\tau_{\nu}\right)\right|^{2 q} \leqq \sum_{\tilde{G}_{l}\left(\tau_{\nu}\right)}\left|1 / c^{*}\left(\tau_{\nu}\right)\right|^{2 q} \leqq M^{\prime}
$$

Therefore

$$
\sum_{W_{2} \in G_{2 l}\left(\tau_{\nu}\right)}\left|W_{2}^{\prime}\left(T_{\nu}^{-1}(w)\right)\right|^{q} \leqq M^{2 q} M^{\prime} / \delta_{3}^{2 q} \quad \text { for } w \in \Omega^{\prime}\left(\tilde{G}_{l}\left(\tau_{0}\right)\right) .
$$

From (19) and (20), we have the desired result.

(3) By modifying the method of Ford ([3], pp. 104-105), we can easily prove the assertion. We omit the proof here.

5-3. We consider the function (16) defined in the proof of Proposition 5 in the remainder of this section. Then

$$
F^{*}\left(\tau_{\nu}, w\right)=\frac{T_{\nu}^{-1^{\prime}}(w)^{q}}{\prod_{i=1}^{u}\left(T_{\nu}^{-1}(w)-a_{i}\left(\tau_{\nu}\right)\right)^{m_{i}} \prod_{j=1}^{v}\left(T_{\nu}^{-1}(w)-b_{j}\left(\tau_{\nu}\right)\right)^{n_{j}}} .
$$

We set $\alpha_{i}^{*}\left(\tau_{\nu}\right)=T_{\nu}\left(a_{i}\left(\tau_{\nu}\right)\right), i=1,2, \cdots, u$ and $b_{j}^{*}\left(\tau_{\nu}\right)=T_{\nu}\left(b_{j}\left(\tau_{\nu}\right)\right), j=1$, $2, \cdots, v$. Since

$$
\begin{aligned}
T_{\nu}^{-1}(w)-a_{i}\left(\tau_{\nu}\right) & =T_{\nu}^{-1}(w)-T_{\nu}^{-1}\left(a_{i}^{*}\left(\tau_{\nu}\right)\right) \\
& =\frac{d_{\nu}\left(1-d_{\nu}\right)\left(w-a_{i}^{*}\left(\tau_{\nu}\right)\right)}{\left(w-d_{\nu}\right)\left(a_{i}^{*}\left(\tau_{\nu}\right)-d_{\nu}\right)},
\end{aligned}
$$

and

$$
T_{\nu}^{-1}(w)-b_{j}\left(\tau_{\nu}\right)=\frac{d_{\nu}\left(1-d_{\nu}\right)\left(w-b_{j}^{*}\left(\tau_{\nu}\right)\right)}{\left(w-d_{\nu}\right)\left(b_{j}^{*}\left(\tau_{\nu}\right)-d_{\nu}\right)_{j}},
$$

we have

$$
\begin{aligned}
f^{*}\left(\tau_{\nu}, w\right) & =\sum_{\gamma^{*} \in \hat{G}_{l}\left(\tau_{\nu}\right)} F^{*}\left(\tau_{\nu}, \gamma^{*}(w)\right) \gamma^{* \prime}(w)^{q} \\
& =\sum \frac{\prod_{i=1}^{u}\left(a_{i}^{*}\left(\tau_{\nu}\right)-d_{\nu}\right)^{n_{i}} \prod_{i=1}^{v}\left(b_{i}^{*}\left(\tau_{\nu}\right)-d_{\nu}\right)^{n_{j}} \gamma^{* \prime}(w)^{q}}{d_{\nu}^{q}\left(1-d_{\nu}\right)^{q} \prod_{i=1}^{u}\left(\gamma^{*}(w)-a_{i}^{*}\left(\tau_{\nu}\right)\right)^{m_{i}} \prod_{j=1}^{v}\left(\gamma^{*}(w)-b_{j}^{*}\left(\tau_{\nu}\right)\right)^{n_{j}}} .
\end{aligned}
$$

By using

$$
a_{i}^{*}\left(\tau_{\nu}\right)-d_{\nu}=d_{\nu}\left(d_{\nu}-1\right) /\left(a_{i}\left(\tau_{\nu}\right)-d_{\nu}\right)
$$




$$
\gamma^{*}(w)-a_{i}^{*}\left(\tau_{\nu}\right)=\frac{d_{\nu}\left(1-d_{\nu}\right)\left(\gamma\left(T_{\nu}^{-1}(w)-a_{i}\left(\tau_{\nu}\right)\right)\right.}{\left(\gamma\left(T_{\nu}^{-1}(w)\right)-d_{\nu}\right)\left(a_{i}\left(\tau_{\nu}\right)-d_{\nu}\right)}
$$

and

$$
\gamma^{* \prime}(w)=\frac{d_{\nu}^{2}\left(1-d_{\nu}\right)^{2}}{\left(\gamma\left(T_{\nu}^{-1}(w)\right)-d_{\nu}\right)^{2}\left(w-d_{\nu}\right)^{2}} \gamma^{\prime}\left(T_{\nu}^{-1}(w)\right),
$$

we have the following, where $\gamma=T_{\nu}^{-1} \gamma^{*} T_{\nu} \in \tilde{G}_{r}\left(\tau_{\nu}\right)$.

LEMMA 4.

$$
\begin{aligned}
f^{*}\left(\tau_{\nu}, w\right)= & \frac{(-1)^{m_{1}+\cdots+m_{u}} d_{\nu}^{2 q}\left(1-d_{\nu}\right)^{q}}{\left(w-d_{\nu}\right)^{2 q}} \\
& \times \sum_{\gamma^{*} \in \tilde{G}_{l}\left(\tau_{\nu}\right)} \frac{1}{\left(\left(T_{\nu}^{-1} \gamma^{*} T_{\nu}\right)\left(T_{\nu}^{-1}(w)\right)-d_{\nu}\right)^{2 q-\left(m_{1}+\cdots+m_{\nu}\right)}} \\
& \times \prod_{j=1}^{v}\left(\frac{b_{j}^{*}\left(\tau_{\nu}\right)-d_{\nu}}{\gamma^{*}(w)-b_{j}^{*}\left(\tau_{\nu}\right)}\right)^{n_{j}} \frac{\left(T_{\nu}^{-1} \gamma^{*} T_{\nu}\right)\left(T_{\nu}^{-1}(w)\right)^{q}}{\prod_{i=1}^{v}\left(\left(T_{\nu}^{-1} \gamma^{*} T_{\nu}\right)\left(T_{\nu}^{-1}(w)\right)-a_{i}\left(\tau_{\nu}\right)\right)^{m_{i}}}
\end{aligned}
$$

5-4. Lemma 5. (1) For every large $\nu$, there exists a positive constant $\delta_{4}$ independent of $\nu$ such that

$$
\left|\gamma\left(T_{\nu}^{-1}(w)\right)-d_{\nu}\right| \geqq \delta_{4}
$$

for all $\gamma=T_{\nu}^{-1} \gamma^{*} T_{\nu} \in G_{1 l}\left(\tau_{\nu}\right)$ and $w \in \Omega^{\prime}\left(\tilde{G}_{l}\left(\tau_{0}\right)\right)$.

(2) For every large $\nu$, there exists a constant $M_{5}$ independent of $\nu$ such that

$$
\prod_{j=1}^{v}\left(b_{j}^{*}\left(\tau_{\nu}\right)-d_{\nu}\right) /\left.\left(\gamma^{*}(w)-b_{j}^{*}\left(\tau_{\nu}\right)\right)\right|^{n_{j}} \leqq M_{5}
$$

for all $\gamma^{*} \in G_{1 l}^{*}\left(\tau_{\nu}\right)$.

Proof. (1) Noting that

$$
\gamma\left(T_{\nu}^{-1}(w)\right) \in \prod_{h=1}^{g_{r}}\left(\left[C_{r, h}\right] \cup\left[C_{r, h}^{\prime}\left(\tau_{\nu}\right)\right]\right) \quad \text { for } \gamma \in G_{1 l}\left(\tau_{\nu}\right)
$$

and that $d_{\nu}$ is sufficiently close to 1 for every large $\nu$, we have the assertion.

(2) Since for every large $\nu$, there exist positive constants $M$ and $\delta$ independent of $\nu$ such that

$$
\left|b_{j}^{*}\left(\tau_{\nu}\right)-d_{\nu}\right| \leqq M \quad(j=1,2, \cdots, v)
$$

and 


$$
\left|\gamma^{*}(w)-b_{j}^{*}\left(\tau_{v}\right)\right| \geqq \delta \quad(j=1,2, \cdots, v),
$$

we have the desired result.

Since

$$
\lim _{\nu \rightarrow \infty} \frac{(-1)^{m_{1}+\cdots+m_{u}} d_{\nu}^{2 q}\left(1-d_{\nu}\right)^{q}}{\left(w-d_{\nu}\right)^{2 q}}=0 \quad \text { for } w \in \Omega^{\prime}\left(\tilde{G}_{l}\left(\tau_{0}\right)\right),
$$

in order to prove Proposition 7 it suffices to show that there exists a constant $M$ independent of $\nu$ such that

$$
\sum_{\gamma \in G_{1 l}\left(\tau_{\nu}\right)} \frac{\gamma^{\prime}\left(T_{\nu}^{-1}(w)\right)^{q}}{\prod_{i=1}^{u}\left(\gamma\left(T_{\nu}^{-1}(w)\right)-a_{i}\left(\tau_{\nu}\right)\right)^{m_{i}}} \leqq M
$$

for every large $\nu$ and $w \in \Omega^{\prime}\left(\tilde{G}_{l}\left(\tau_{0}\right)\right)$.

5-5. Lemma 6. Suppose $a_{i}\left(\tau_{\nu}\right)$ is a distinguished point. Then for every large $\nu$, there exists a positive constant $\delta$ independent of $\nu$ such that

$$
\left|\gamma\left(T_{\nu}^{-1}(w)\right)-a_{i}\left(\tau_{\nu}\right)\right| \geqq \delta_{5} \quad \text { for all } \gamma \in G_{1 l}\left(\tau_{\nu}\right) .
$$

The proof is obvious and so we omit it here.

By the above lemma, from now on, we assume that each $a_{i}\left(\tau_{\nu}\right), i=$ $1,2, \cdots, u$, is not a distinguished point. Furthermore we assume that $a_{i}\left(\tau_{\nu}\right)(i=1,2, \cdots, u)$ is the repelling fixed point of $A_{r, h(i)}\left(\tau_{\nu}, z\right)$. For the other cases, we can treat similarly to this one.

We decompose (23) as follows:

$$
\begin{aligned}
\sum_{G_{1 l}\left(\tau_{\nu}\right)} & \frac{\gamma^{\prime}\left(T_{\nu}^{-1}(w)\right)^{q}}{\prod_{i=1}^{u}\left(\gamma\left(T_{\nu}^{-1}(w)\right)-a_{i}\left(\tau_{\nu}\right)\right)^{m_{i}}} \\
= & \sum_{k=1}^{u} \sum_{G_{r}, h_{h(k)}\left(\tau_{\nu}\right)} \frac{\gamma^{\prime}\left(T_{\nu}^{-1}(w)\right)^{q}}{\prod_{i=1}^{u}\left(\gamma\left(T_{\nu}^{-1}(w)\right)-a_{i}\left(\tau_{\nu}\right)\right)^{m_{i}}} \\
& \quad+\underset{G_{1 l}\left(\tau_{\nu}\right) \backslash \cup_{k=1}^{u} G_{r, h(k)}\left(\tau_{\nu}\right)}{\prod_{i=1}^{u}\left(\gamma\left(T_{\nu}^{-1}(w)\right)-a_{i}\left(\tau_{\nu}\right)\right)^{m_{i}}} .
\end{aligned}
$$

Lemma 7. For every large $\nu$, there exists a positive constant $\delta_{6}$ independent of $\nu$ such that

$$
\left|1 /\left(\gamma\left(T_{\nu}^{-1}(w)\right)-a_{i}\left(\tau_{\nu}\right)\right)\right| \geqq \delta_{6}
$$

for all $\gamma \in G_{11}\left(\tau_{\nu}\right) \backslash \bigcup_{k=1}^{u} G_{r, h(k)}\left(\tau_{\nu}\right)$.

From Lemmas 3 and 7, we have the following.

Lemma 8. For every large $\nu$, there exists a constant $M_{6}$ independent of $\nu$ such that 


$$
\sum_{G_{1 l}\left(\tau_{\nu}\right) \backslash \cup_{k=1}^{u} G_{r, h(k)}\left(\tau_{\nu}\right)} \frac{\gamma^{\prime}\left(T_{\nu}^{-1}(w)\right)^{q}}{\prod_{i=1}^{u}\left(\gamma\left(T_{\nu}^{-1}(w)\right)-a_{i}\left(\tau_{\nu}\right)\right)^{m_{i}}} \leqq M_{6} .
$$

5-6. Next we consider

$$
\sum_{G_{r, h(k)}\left(\tau_{\nu}\right)} \frac{\gamma^{\prime}\left(T_{\nu}^{-1}(w)\right)^{q}}{\prod_{i=1}^{u}\left(\gamma\left(T_{\nu}^{-1}(w)\right)-a_{i}\left(\tau_{\nu}\right)\right)^{m_{i}}}, \quad k=1,2, \cdots, u .
$$

For each $k=1,2, \cdots, u$, we easily see the following.

Lemma 9. For every large $\nu$, there exists a positive constant $\delta_{7}$ independent of $\nu$ such that

$$
\sum_{\substack{i=1 \\ i \neq k}}^{u}\left|\gamma\left(T_{\nu}^{-1}(w)\right)-a_{i}\left(\tau_{\nu}\right)\right| \geqq \delta_{7} \quad \text { for } \gamma \in G_{r, h(k)}\left(\tau_{\nu}\right)
$$

Thus in order to show the boundedness of (24), it suffices to show by Lemma 3 that there exists a constant $M$ independent of $\nu$ such that

$$
\sum_{G_{r, h(k)}\left(\tau_{\nu}\right)}\left|\frac{\gamma^{\prime}\left(T_{\nu}^{-1}(w)\right)^{q}}{\left(\gamma\left(T_{\nu}^{-1}(w)\right)-a_{k}\left(\tau_{\nu}\right)\right)^{m_{k}}}\right| \leqq M
$$

for every large $\nu$ and for $k=1,2, \cdots, u$.

We set

$$
f_{1 k}\left(\tau_{\nu}, w\right)=\sum_{G_{r, h(k)}\left(\tau_{\nu}\right)} \frac{\gamma^{\prime}\left(T_{\nu}^{-1}(w)\right)^{q}}{\left(\gamma\left(T_{\nu}^{-1}(w)\right)-a_{k}\left(\tau_{\nu}\right)\right)^{m_{k}}}
$$

Here we introduce the Möbius transformation defined by

$$
\zeta=S_{\nu}(z)=\frac{z-a_{k}\left(\tau_{\nu}\right)}{z-a_{k}^{\prime}\left(\tau_{\nu}\right)} \frac{1-a_{k}^{\prime}\left(\tau_{\nu}\right)}{1-a_{k}\left(\tau_{\nu}\right)}
$$

where $a_{k}^{\prime}\left(\tau_{\nu}\right)$ is the attracting fixed point of $A_{r, h(k)}\left(\tau_{\nu}, z\right)$. Then we have

$$
z=S_{\nu}^{-1}(\zeta)=\frac{\left(1-a_{k}\left(\tau_{\nu}\right)\right) a_{k}^{\prime}\left(\tau_{\nu}\right) \zeta-a_{k}\left(\tau_{\nu}\right)\left(1-a_{k}^{\prime}\left(\tau_{\nu}\right)\right)}{\left(1-a_{k}\left(\tau_{\nu}\right)\right) \zeta-\left(1-a_{k}^{\prime}\left(\tau_{\nu}\right)\right)}
$$

and

$$
S_{\nu}^{\prime}(z)=\frac{1-a_{k}^{\prime}\left(\tau_{\nu}\right)}{1-a_{k}\left(\tau_{\nu}\right)} \frac{a_{k}\left(\tau_{\nu}\right)-a_{k}^{\prime}\left(\tau_{\nu}\right)}{\left(z-a_{k}^{\prime}\left(\tau_{\nu}\right)\right)^{2}}
$$

We set $G_{r, h(k)}\left(\tau_{\nu}\right)=S_{\nu}^{-1} G_{r, h(k)}\left(\tau_{\nu}\right) S_{\nu}$.

By simple computation, we obtain 
Lemma 10.

$$
\begin{aligned}
f_{l k}\left(\tau_{\nu}, w\right)= & \sum_{\check{r} \in \check{G}_{r, h(k)}\left(\tau_{\nu}\right)} \frac{(-1)^{q}\left\{\left(1-a_{k}\left(\tau_{\nu}\right)\right)\left(1-a_{k}^{\prime}\left(\tau_{\nu}\right)\right)\left(a_{k}^{\prime}\left(\tau_{\nu}\right)-a_{k}\left(\tau_{\nu}\right)\right)\right\}^{q-m_{k}}}{\left\{\left(1-a_{k}\left(\tau_{\nu}\right)\right) \check{\gamma}^{\prime}\left(S_{\nu} T_{\nu}^{-1}(w)\right)-\left(1-a_{k}^{\prime}\left(\tau_{\nu}\right)\right)\right\}^{2 q-m_{k}}} \\
& \times \frac{\check{\gamma}^{\prime}\left(S_{\nu} T_{\nu}^{-1}(w)\right)^{q}}{\check{\gamma}\left(S_{\nu} T_{\nu}^{-1}(w)\right)^{m_{k}}},
\end{aligned}
$$

where $\check{\gamma}=S_{\nu}^{-1} \gamma S_{\nu}$ with $\gamma \in G_{r, h(k)}\left(\tau_{\nu}\right)$.

By noting that $\left(1-a_{k}^{\prime}\left(\tau_{\nu}\right)\right) /\left(1-a_{k}\left(\tau_{\nu}\right)\right)=S_{\nu}(\infty) \in S_{\nu}\left(\omega_{r}\left(\tau_{\nu}\right)\right)$, we see the following lemma.

Lemma 11. For every large $\nu$, there exists a constant $M_{7}$ independent of $\nu$ such that

$$
\left|\frac{(-1)^{q}\left(1-a_{k}^{\prime}\left(\tau_{\nu}\right)\right)^{q-m_{k}}\left(a_{k}^{\prime}\left(\tau_{\nu}\right)-a_{k}\left(\tau_{\nu}\right)\right)^{q-m_{k}}}{\left(1-a_{k}\left(\tau_{\nu}\right)\right)^{q}\left\{\check{\gamma}\left(S_{\nu} T_{\nu}^{-1}(w)\right)-\left(1-a_{k}^{\prime}\left(\tau_{\nu}\right)\right) /\left(1-a_{k}\left(\tau_{\nu}\right)\right)\right\}^{2 q-m_{k}}}\right| \leqq M_{7}
$$

for all $\check{\gamma} \in \check{G}_{r, h(k)}\left(\tau_{\nu}\right)$.

5-7. Any element $\check{\gamma} \in \check{G}_{r, h(k)}\left(\tau_{\nu}\right)$ is represented as $\check{\gamma}\left(\tau_{\nu}, \zeta\right)=\check{A}_{r, h(k)}^{-m}\left(\check{B}\left(\tau_{\nu}, \zeta\right)\right)$ $(m>0)$, where $\check{B} \in S_{\nu}^{-1} \bar{G}_{r, h(k)}\left(\tau_{\nu}\right) S_{\nu}$, namely if we write $\check{B}=\check{B}_{1} \check{B}_{2} \cdots \check{B}_{n}$, then $\check{B}_{1} \neq A_{r, h(k)}^{ \pm 1}\left(\tau_{\nu}, z\right)$. Since $\check{A}_{r, h(k)}^{-1}\left(\tau_{\nu}, \zeta\right)=t_{h(k)}\left(\tau_{\nu}\right) \zeta$ with $0<\left|t_{h(k)}\left(\tau_{\nu}\right)\right|<1$ and $\lim _{\nu \rightarrow \infty} t_{h(k)}\left(\tau_{\nu}\right) \neq 0$, where $t_{h(k)}^{-1}\left(\tau_{\nu}\right)$ is the multiplier of $A_{r, h(k)}\left(\tau_{\nu}, z\right)$, we obtain the following

LEMMA 12.

$$
\begin{aligned}
\sum_{\check{G}_{r, h(k)}\left(\tau_{\nu}\right)} & \frac{\check{\gamma}^{\prime}\left(S_{\nu} T_{\nu}^{-1}(w)\right)^{q}}{\check{\gamma}\left(S_{\nu} T_{\nu}^{-1}(w)\right)^{m_{k}}} \\
& =\frac{t_{h(k)}\left(\tau_{\nu}\right)^{q-m_{k}}}{1-t_{h(k)}\left(\tau_{\nu}\right)^{q-m_{k}}} \sum_{\check{G}_{r, h(k)}\left(\tau_{\nu}\right)} \frac{\check{B}^{\prime}\left(S_{\nu} T_{\nu}^{-1}(w)\right)^{q}}{\check{B}^{\prime}\left(S_{\nu} T_{\nu}^{-1}(w)\right)^{m_{k}}},
\end{aligned}
$$

where $\overline{\check{G}}_{r, h(k)}\left(\tau_{\nu}\right)=S_{\nu} \bar{G}_{r, h(k)}\left(\tau_{\nu}\right) S_{\nu}^{-1}$.

The following Lemmas 13, 14 and 15 are easily seen.

Lemma 13. For every large $\nu$, there exists a positive constant $\delta_{3}$ independent of $\nu$ such that

$$
\left|\check{B}\left(S_{\nu} T_{\nu}^{-1}(w)\right)\right| \geqq \delta_{8}
$$

for all $\check{B} \in \check{G}_{r, h(k)}\left(\tau_{\nu}\right)$ and for $w \in \Omega^{\prime}\left(\tilde{G}_{l}\left(\tau_{0}\right)\right)$. 
LEMma 14.

$$
\begin{aligned}
& \quad \sum_{\bar{\sigma}_{r, h(k)}\left(\tau_{\nu}\right)} \check{B}^{\prime}\left(S_{\nu} T_{\nu}^{-1}(w)\right)^{q} \\
& \quad=\sum_{B \in \tilde{G}_{r, h(k)}\left(\tau_{\nu}\right)}\left(\left(T_{\nu}^{-1}(w)-a_{k}^{\prime}\left(\tau_{\nu}\right)\right) /\left(B\left(T_{\nu}^{-1}(w)\right)-a_{k}^{\prime}\left(\tau_{\nu}\right)\right)\right)^{2 q} B^{\prime}\left(T_{\nu}^{-1}(w)\right)^{q} .
\end{aligned}
$$

Lemma 15. For every large $\nu$, there exists a constant $M_{8}$ independent of $\nu$ such that

$$
\left|\left(T_{\nu}^{-1}(w)-a_{k}^{\prime}\left(\tau_{\nu}\right)\right) /\left(B\left(T_{\nu}^{-1}(w)\right)-a_{k}^{\prime}\left(\tau_{\nu}\right)\right)\right| \leqq M_{8}
$$

for all $B \in \bar{G}_{r, h(k)}\left(\tau_{\nu}\right)$ and for $w \in \Omega^{\prime}\left(\tilde{G}_{l}\left(\tau_{0}\right)\right)$.

By Lemmas 3, 9, 10, 11, 12, 13, 14, 15, we have

Lemma 16. For every large $\nu$, there exists a constant $M_{9}$ independent of $\nu$ such that

$$
\sum_{k=1}^{u} \sum_{\sigma_{r, h(k)}\left(\tau_{\nu}\right)}\left|\frac{\gamma^{\prime}\left(T_{\nu}^{-1}(w)\right)^{q}}{\prod_{i=1}^{u}\left(\gamma\left(T_{\nu}^{-1}(w)\right)-a_{i}\left(\tau_{\nu}\right)\right)^{m_{k}}}\right| \leqq M_{9} .
$$

Lemmas 4, 5, 7, 16 and (22) contain Proposition 7.

5-8. Next we will prove Proposition 8. We recall that

$$
\begin{aligned}
f_{2}^{*}\left(\tau_{\nu}, w\right)= & \frac{\prod_{i=1}^{u}\left(a_{i}^{*}\left(\tau_{\nu}\right)-d_{\nu}\right)^{m_{i}} \prod_{j=1}^{v}\left(b_{j}^{*}\left(\tau_{\nu}\right)-d_{\nu}\right)^{n_{j}}}{d_{\nu}^{q}\left(1-d_{\nu}\right)^{q}} \\
& \times \sum_{\gamma^{*} \in G_{2 l}^{*}\left(\tau_{\nu}\right)} \frac{\gamma^{* \prime}(w)^{q}}{\prod_{i=1}^{u}\left(\gamma^{*}(w)-a_{i}^{*}\left(\tau_{\nu}\right)\right)^{m_{i}} \prod_{j=1}^{v}\left(\gamma^{*}(w)-b_{j}^{*}\left(\tau_{\nu}\right)\right)^{n_{j}}} .
\end{aligned}
$$

LEMMA 17.

$$
\lim _{\nu \rightarrow \infty} \frac{\prod_{i=1}^{u}\left(a_{1}^{*}\left(\tau_{\nu}\right)-d_{\nu}\right)^{m_{i}} \prod_{j=1}^{v}\left(b_{j}^{*}\left(\tau_{\nu}\right)-d_{\nu}\right)^{n_{j}}}{d_{\nu}^{q}\left(1-d_{\nu}\right)^{q}}=0 .
$$

Proof. We have

$$
\lim _{\nu \rightarrow \infty} d_{\nu}=1, \quad a_{i}^{*}\left(\tau_{\nu}\right)-d_{\nu}=\left(d_{\nu}\left(d_{\nu}-1\right)\right) /\left(a_{i}\left(\tau_{\nu}\right)-d_{\nu}\right)
$$

$(i=1,2, \cdots, u)$ and, as we showed in pp. 16, 17,

$$
\begin{aligned}
& \lim _{\nu \rightarrow \infty}\left(b_{j}^{*}\left(\tau_{\nu}\right)-d_{\nu}\right)=b_{j}^{*}\left(\tau_{0}\right)-1 \neq \infty \\
& \lim _{\nu \rightarrow \infty}\left(a_{i}\left(\tau_{\nu}\right)-d_{\nu}\right)=a_{0}\left(\tau_{0}\right)-1 \neq 0 .
\end{aligned}
$$

Accordingly, the left hand side of (27) is equal to 


$$
\frac{\prod_{j=1}^{v}\left(b_{j}^{*}\left(\tau_{0}\right)-1\right)^{n_{j}}}{\prod_{i=1}^{u}\left(a_{i}\left(\tau_{0}\right)-1\right)^{m_{i}}} \lim _{\nu \rightarrow \infty} \frac{(-1)^{m_{1}+\cdots+m_{u}}\left(1-d_{\nu}\right)^{m_{1}+\cdots+m_{u}}}{\left(1-d_{\nu}\right)^{q}} .
$$

Since $m_{1}+\cdots+m_{u} \geqq q+1$, we have the desired result.

Noting that $\lim _{\nu \rightarrow \infty} a_{i}^{*}\left(\tau_{\nu}\right)=1(i=1,2, \cdots, u)$, we easily see the following.

Lemma 18. For every large $\nu$, there exists a positive constant $\delta_{8}$ independent of $\nu$ such that

$$
\left|\gamma^{*}(w)-a_{i}^{*}\left(\tau_{\nu}\right)\right| \geqq \delta_{8}
$$

for each $i=1,2, \cdots, u$ and for all $\gamma^{*} \in G_{2 l}^{*}\left(\tau_{\nu}\right)$.

On the other hand, by the same way as in the proof of Proposition 7, we can show the following lemma.

Lemma 19. For every large $\nu$, there exists a constant $M_{10}$ independent of $\nu$ such that

$$
\sum_{G_{2 l^{*}}\left(\tau_{\nu}\right)}\left|\frac{\gamma^{* \prime}(w)^{q}}{\prod_{j=1}^{v}\left(\gamma^{*}(w)-b_{j}^{*}\left(\tau_{\nu}\right)\right)^{n_{j}}}\right| \leqq M_{10}
$$

for $w \in \Omega^{\prime}\left(\tilde{G}_{l}\left(\tau_{0}\right)\right)$.

By Lemmas 17, 18 and 19, we prove Proposition 8.

\section{§6. Continuity of functions $\theta_{s, \pi}, \psi_{j}$ and $\psi_{s, \sigma}$ (continued from §4)}

Proposition 9. Under the same assumption as in Proposition 5, if $F$ is one of the functions $\Phi_{s, h}\left(\tau_{\nu}, z\right) \in N_{s}\left(\tau_{\nu}\right)$ such that $\Phi\left(\tau_{0}, z\right) \in N_{s}\left(\tau_{0}\right)$ with $s \in$ $W_{r}(L)$, then (13) and (17) are satisfied. Here $f$ and $f^{*}$ are defined from $F$ in §4-2.

Proof. It suffices to consider the following function:

$$
F\left(\tau_{\nu}, z\right)=\frac{\left(p_{s, h}\left(\tau_{\nu}\right)-q_{s, h}\left(\tau_{\nu}\right)\right)^{q}}{\left(z-p_{s, h}\left(\tau_{\nu}\right)\right)^{q}\left(z-q_{s, h}\left(\tau_{\nu}\right)\right)^{q}},
$$

$h=1,2, \cdots, g_{r}+k_{r}$. Let

$$
\tilde{G}_{r}\left(\tau_{\nu}\right)=\hat{G}_{r, h}\left(\tau_{\nu}\right) \gamma_{0}+\hat{G}_{r, h}\left(\tau_{\nu}\right) \gamma_{1}+\hat{G}_{r, h}\left(\tau_{\nu}\right) \gamma_{2}+\cdots,
$$

where $\hat{G}_{r, h}\left(\tau_{\nu}\right)=\left\langle A_{r, h}\left(\tau_{\nu}, z\right)\right\rangle$ and $\gamma_{0}=\mathrm{id}$., $\gamma_{1}, \gamma_{2}, \ldots$ is a complete list of the right coset representatives of $\tilde{G}_{r}\left(\tau_{\nu}\right)$ modulo $\hat{G}_{r, h}\left(\tau_{\nu}\right)$. Set $F^{*}\left(\tau_{\nu}, w\right)=$ $F\left(\tau_{\nu}, T_{\nu}^{-1}(w)\right) T_{\nu}^{-1^{\prime}}(w)^{q}$ and $f^{*}\left(\tau_{\nu}, w\right)=\sum_{n=0}^{\infty} F^{*}\left(\tau_{\nu}, \gamma_{n}^{*}(w)\right) \gamma_{n}^{* \prime}(w)^{q}$. Then $f^{*}\left(\tau_{\nu}, w\right)$ 
$=f\left(\tau_{\nu}, T_{\nu}^{-1}(w)\right) T_{\nu}^{-1^{\prime}}(w)^{q}$. Since $F^{*}\left(\tau_{0}, w\right)=0$ for $w \in \Omega^{\prime}\left(\tilde{G}_{l}\left(\tau_{0}\right)\right)$ by Lemma 1 , $f^{*}\left(\tau_{0}, w\right)=0$. Thus it suffices to show that

$$
\lim _{\nu \rightarrow \infty} f\left(\tau_{\nu}, z\right)=f\left(\tau_{0}, z\right) \quad \text { for } z \in \Omega^{\prime}\left(\tilde{G}_{r}\left(\tau_{0}\right)\right)
$$

and

$$
\lim _{\nu \rightarrow \infty} f^{*}\left(\tau_{\nu}, w\right)=0 \quad \text { for } w \in \Omega^{\prime}\left(\tilde{G}_{l}\left(\tau_{0}\right)\right) .
$$

By a similar method to the proof of Proposition 6, we can show (29). Here we will show only (28). Let

$$
\hat{G}_{r}\left(\tau_{\nu}\right)=\hat{G}_{r, h}\left(\tau_{\nu}\right) \hat{\gamma}_{0}+\hat{G}_{r, h}\left(\tau_{\nu}\right) \hat{\gamma}_{1}+\hat{G}_{r, h}\left(\tau_{\nu}\right) \hat{\gamma}_{2}+\cdots,
$$

where $\gamma_{0}=\mathrm{id}$., $\hat{\gamma}_{1}, \hat{\gamma}_{2}, \ldots$ is a complete list of the right coset representatives of $\hat{G}_{r}\left(\tau_{\nu}\right)$ modulo $\hat{G}_{r, h}\left(\tau_{\nu}\right)$. We denote by $E$ and $\hat{E}$ the sets $\left\{\gamma_{0}, \gamma_{1}, \gamma_{2}, \cdots\right\}$ and $\left\{\hat{\gamma}_{0}, \hat{\gamma}_{1}, \hat{\gamma}_{2}, \cdots\right\}$, respectively. We may assume that $E \supset \hat{E}$ and

$$
E=\hat{E} \tilde{\gamma}_{0} \cup \hat{E} \tilde{\gamma}_{1} \cup \hat{E} \tilde{\gamma}_{2} \cup \cdots,
$$

with mutually disjoint $\hat{E} \tilde{\gamma}_{i}$.

We set

$$
\hat{F}\left(\tau_{\nu}, z\right)=\sum_{n=0}^{\infty} F\left(\tau_{\nu}, \hat{\gamma}_{n}(z)\right) \hat{\gamma}_{n}^{\prime}(z)^{q}
$$

By the same way as before, we see that the series (30) satisfies the following:

(i) $\hat{F}\left(\tau_{0}, z\right)=f\left(\tau_{0}, z\right)$,

(ii) the series (30) converges absolutely, and

(iii) $F(\tau, z)$ is holomorphic on $(\tau, z) \in \sum_{t=0}^{2 q-3} \widetilde{\mho}_{t} \mathfrak{S}_{g}^{I, L}(\Sigma)$.

We have the following absolutely convergent expansion

$$
f(\tau, z)=\sum_{n=0}^{\infty} \hat{F}\left(\tau, \tilde{\gamma}_{n}(z)\right) \tilde{\gamma}_{n}^{\prime}(z)^{q} .
$$

We set

$$
R_{\nu}(z)=\sum_{n=1}^{\infty} \hat{F}\left(\tau_{\nu}, \tilde{\gamma}_{n}(z)\right) \tilde{\gamma}_{n}^{\prime}(z)^{q}
$$

Let $\omega_{r}\left(\tau_{\nu}\right)$ and $\hat{\omega}_{r}\left(\tau_{\nu}\right)$ be the fundamental domains for $\tilde{G}_{r}\left(\tau_{\nu}\right)$ and $\hat{G}_{r}\left(\tau_{\nu}\right)$, respectively which we constructed in the proof of Proposition 5. Let $\omega_{r, h}\left(\tau_{\nu}\right)$ be the fundamental domain for $\hat{G}_{r, h}\left(\tau_{\nu}\right)$ bounded by $C_{r, h}$ and $C_{r, h}^{\prime}\left(\tau_{\nu}\right)$. By Proposition 1, we have 


$$
\begin{aligned}
& \iint_{\hat{\omega}_{r}\left(\tau_{\nu}\right)}\left|\hat{F}\left(\tau_{\nu}, z\right)\right| \lambda\left(\tau_{\nu}, z\right)^{2-q} d x d y \\
& \quad \leqq \iint_{\omega_{r, h}\left(\tau_{\nu}\right)}\left|F\left(\tau_{\nu}, z\right)\right| \lambda\left(\tau_{\nu}, z\right)^{2-q} d x d y .
\end{aligned}
$$

Then the right hand side tends to

$$
\iint_{\omega_{r, h\left(\tau_{0}\right)}}\left|F\left(\tau_{0}, z\right)\right| \lambda\left(\tau_{0}, z\right)^{2-q} d x d y<+\infty
$$

as $\nu \rightarrow \infty$, where $\omega_{r, h}\left(\tau_{0}\right)$ is the fundamental domain for $\hat{G}_{r, h}\left(\tau_{0}\right)=\left\langle A_{r, h}\left(\tau_{0}, z\right)\right\rangle$ bounded by $C_{r, h}$ and $C_{r, h}^{\prime}\left(\tau_{0}\right)=A_{r, h}\left(\tau_{0}, C_{r, h}\right)$ and $\lambda\left(\tau_{0}, z\right)|d z|$ is the Poincaré metric on $\Omega^{\prime}\left(\tilde{G}_{r}\left(\tau_{0}\right)\right)$. Hence for properly chosen fixed circle $C$ surrounding the point 1 ,

$$
\max _{z \in C}\left|F\left(\tau_{\nu}, z\right)\right|=O(1), \nu \longrightarrow \infty .
$$

Since $F\left(\tau_{\nu}, z\right)$ is holomorphic in the interior to $C,\left|\hat{F}\left(\tau_{\nu}, z\right)\right| \lambda\left(\tau_{\nu}, z\right)^{2-q}$ is uniformly bounded, i.e., there exists a constant $M$ independent of $\nu$ such that

$$
\left|\hat{F}\left(\tau_{\nu}, z\right)\right| \lambda\left(\tau_{\nu}, z\right)^{2-q} \leqq M
$$

Thus by a similar method to the proof of Proposition 5, we have

$$
\iint_{\omega_{r}\left(\tau_{\nu}\right)}\left|R_{\nu}(z)\right| \lambda\left(\tau_{\nu}, z\right)^{2-q} d x d y \leqq M \text { Area }\left(\hat{\omega}_{r}\left(\tau_{\nu}\right) \backslash \omega_{r}\left(\tau_{\nu}\right)\right) .
$$

Since Area $\left(\hat{\omega}_{r}\left(\tau_{\nu}\right) \mid \omega_{r}\left(\tau_{\nu}\right)\right) \rightarrow 0$ as $\nu \rightarrow \infty$, we have $\lim _{\nu \rightarrow \infty} R_{\nu}(z)=0$ for $z \in$ $\Omega^{\prime}\left(\tilde{G}_{r}\left(\tau_{0}\right)\right)$.

6-2. Proposition 10. Under the same assumption as in Proposition 5 , if $F$ is one of the functions $\Theta_{s, \pi}\left(\tau_{\nu}, z\right) \in M_{s}\left(\tau_{\nu}\right)$ such that $\Theta_{s, \pi}\left(\tau_{0}, z\right) \in L_{s}\left(\tau_{0}\right)$ with $s \in W_{r}(L)$, then (13) and (17) are satisfied, where $f$ and $f^{*}$ are defined from $F$ in $\S 4-2$.

Proof. It suffices to consider the following function:

$$
\begin{aligned}
& F\left(\tau_{\nu}, z\right)=1 /\left(\prod_{i=1}^{u}\left(z-a_{i}\left(\tau_{\nu}\right)\right)^{m_{i}} \prod_{j=1}^{v}\left(z-b_{j}\left(\tau_{\nu}\right)\right)^{n_{j}}\right), \\
& F\left(\tau_{0}, z\right)=\lim _{\nu \rightarrow \infty} F\left(\tau_{\nu}, z\right)
\end{aligned}
$$

with $a_{i}\left(\tau_{\nu}\right) \in \hat{P}_{r}\left(\tau_{\nu}\right)(i=1,2, \cdots, u), b_{j}\left(\tau_{\nu}\right) \in \hat{P}_{l}\left(\tau_{\nu}\right)(j=1,2, \cdots, v), m_{1}+\cdots$ - $m_{u}=n_{1}+\cdots+n_{v}=q, u \geqq 2, v \geqq 2, m_{i} \geqq 1$ and $n_{j} \geqq 1$, where $a_{i}\left(\tau_{\nu}\right)$ and $b_{j}\left(\tau_{\nu}\right)$ are selected as in $\S 3-2$, pp. 87-88.

We have 


$$
\begin{aligned}
& F\left(\tau_{0}, z\right)=1 /\left(\prod_{i=1}^{u}\left(z-a_{i}\left(\tau_{0}\right)\right)^{m_{i}}(z-1)^{q}\right), \\
& \lim _{\nu \rightarrow \infty} F^{*}\left(\tau_{\nu}, w\right)=(-1)^{q} \frac{\prod_{j=1}^{v}\left(b_{j}^{*}\left(\tau_{0}\right)-1\right)^{n_{j}}}{\prod_{i=1}^{u}\left(a_{i}\left(\tau_{0}\right)-1\right)^{m_{i}}(w-1)^{q} \prod_{j=1}^{v}\left(w-b_{j}^{*}\left(\tau_{0}\right)\right)^{n_{j}}} \\
& f\left(\tau_{0}, z\right)=\sum_{\gamma \in \tilde{\sigma}_{r}\left(\tau_{0}\right)} F\left(\tau_{0}, \gamma(z)\right) \gamma^{\prime}(z)^{q}
\end{aligned}
$$

where $b_{j}^{*}\left(\tau_{\nu}\right)=T_{\nu}\left(b_{j}\left(\tau_{\nu}\right)\right)$ and $b_{j}^{*}\left(\tau_{0}\right)=\lim _{\nu \rightarrow \infty} b_{j}^{*}\left(\tau_{\nu}\right)$. Furthermore if we set $F^{*}\left(\tau_{0}, w\right)=\lim _{\nu \rightarrow \infty} F^{*}\left(\tau_{\nu}, w\right)$, then

$$
f^{*}\left(\tau_{0}, w\right)=\sum_{\gamma^{*} \in \tilde{\sigma}_{l}\left(\tau_{0}\right)} F^{*}\left(\tau_{0}, \gamma^{*}(w)\right) \gamma^{* \prime}(w)^{q}
$$

We will show that

$$
\lim _{\nu \rightarrow \infty} f\left(\tau_{\nu}, z\right)=f\left(\tau_{0}, z\right) \quad \text { for } z \in \Omega^{\prime}\left(\tilde{G}_{r}\left(\tau_{0}\right)\right)
$$

and

$$
\lim _{\nu \rightarrow \infty} f^{*}\left(\tau_{\nu}, w\right)=f^{*}\left(\tau_{0}, w\right) \quad \text { for } w \in \Omega^{\prime}\left(\tilde{G}_{l}\left(\tau_{0}\right)\right)
$$

We can prove (32) by combining the methods of proofs of (31) and Proposition 6. Therefore we will prove only (31) here.

We decompose the group $\tilde{G}_{r}\left(\tau_{\nu}\right)$ into the following two sets:

$$
\begin{aligned}
G_{1 r}\left(\tau_{\nu}\right)=\left\{\gamma \in \tilde{G}_{r}\left(\tau_{\nu}\right) \mid \gamma=\mathrm{id} . \text { or } \gamma(z) \in \bigcup_{h=1}^{g_{r}}\left(\left[C_{r, h}\right] \cup\left[C_{r, h}^{\prime}\left(\tau_{\nu}\right)\right]\right)\right. \\
\\
\left.\quad \text { for some } z \in \omega_{r}\left(\tau_{\nu}\right)\right\}
\end{aligned}
$$

and

$$
G_{2 r}\left(\tau_{\nu}\right)=\tilde{G}_{r}\left(\tau_{\nu}\right) \backslash G_{1 r}\left(\tau_{\nu}\right)
$$

We note that $G_{1 r}\left(\tau_{\nu}\right)=G_{1 l}\left(\tau_{\nu}\right) \cup\{$ id. $\}$ and $G_{2 r}\left(\tau_{\nu}\right)=G_{2 l}\left(\tau_{\nu}\right) \backslash\{$ id. $\}$. We write

$$
\begin{aligned}
f\left(\tau_{\nu}, z\right) & =\sum_{\gamma \in G_{1 r}\left(\tau_{\nu}\right)} F\left(\tau_{\nu}, \gamma(z)\right) \gamma^{\prime}(z)^{q}+\sum_{r \in G_{2 r}\left(\tau_{\nu}\right)} F\left(\tau_{\nu}, \gamma(z)\right) \gamma^{\prime}(z)^{q} \\
& =f_{1}\left(\tau_{\nu}, z\right)+f_{2}\left(\tau_{\nu}, z\right) .
\end{aligned}
$$

We set $a_{i}^{*}\left(\tau_{\nu}\right)=T_{\nu}\left(a_{i}\left(\tau_{\nu}\right)\right)$ and $G_{2 r}^{*}\left(\tau_{\nu}\right)=T_{\nu} G_{2 r}\left(\tau_{\nu}\right) T_{\nu}^{-1}$. Setting

$$
\tilde{F}^{*}\left(\tau_{\nu}, w\right)=1 /\left(\prod_{i=1}^{u}\left(w-a_{i}^{*}\left(\tau_{\nu}\right)\right)^{m_{i}} \prod_{j=1}^{v}\left(w-b_{j}^{*}\left(\tau_{\nu}\right)\right)^{n_{j}}\right),
$$

we have

$$
f_{2}\left(\tau_{\nu}, z\right)=\sum_{\left.\tau^{*} \in G_{2 \tau^{*}}^{*} \tau_{\nu}\right)} F^{*}\left(\tau_{\nu}, \gamma^{*}\left(T_{\nu}(z)\right)\right) \gamma^{* \prime}\left(T_{\nu}(z)\right)^{q} T_{\nu}^{\prime}(z)^{q}
$$




$$
\begin{aligned}
= & \frac{\prod_{i=1}^{u}\left(a_{i}^{*}\left(\tau_{\nu}\right)-d_{\nu}\right)^{m_{i}} \prod_{j=1}^{v}\left(b_{j}^{*}\left(\tau_{\nu}\right)-d_{\nu}\right)^{n_{j}}}{d_{\nu}^{q}\left(1-d_{\nu}\right)^{q}} \\
& \times\left(\sum_{G_{2 r}^{*}\left(\tau_{\nu}\right)} \tilde{F}^{*}\left(\tau_{\nu}, \gamma^{*}\left(T_{\nu}(z)\right) \gamma^{* \prime}\left(T_{\nu}(z)\right)^{q}\right) T_{\nu}^{\prime}(z)^{q} .\right.
\end{aligned}
$$

Since

$$
\begin{aligned}
& \prod_{i=1}^{u}\left(a_{i}^{*}\left(\tau_{\nu}\right)-d_{\nu}\right)^{m_{i}} \prod_{j=1}^{v}\left(b_{j}^{*}\left(\tau_{\nu}\right)-d_{\nu}\right)^{n_{j}} \\
& =\frac{d_{\nu}^{q}\left(d_{\nu}-1\right)^{q} \prod_{j=1}^{v}\left(b_{j}^{*}\left(\tau_{\nu}\right)-d_{\nu}\right)^{n_{j}}}{\prod_{i=1}^{u}\left(a_{i}\left(\tau_{\nu}\right)-d_{\nu}\right)^{m_{i}}},
\end{aligned}
$$

we have

$$
\begin{aligned}
f_{2}\left(\tau_{\nu}, z\right)= & \frac{(-1)^{q} \prod_{j=1}^{v}\left(b_{j}^{*}\left(\tau_{\nu}\right)-d_{\nu}\right)^{n_{j}}}{\prod_{i=1}^{u}\left(a_{i}\left(\tau_{\nu}\right)-d_{\nu}\right)^{m_{i}}} \\
& \times\left(\sum_{G_{2 r}^{*}\left(\tau_{\nu}\right)} \tilde{F}^{*}\left(\tau_{\nu}, \gamma^{*}\left(T_{\nu}(z)\right)\right) \gamma^{* \prime}\left(T_{\nu}(z)\right)^{q}\right) T_{\nu}^{\prime}(z)^{q} .
\end{aligned}
$$

By a similar method to the proof of Proposition 7, we have

$$
\lim _{\nu \rightarrow \infty} \sum_{G_{2 r}^{*}\left(\tau_{\nu}\right)} \tilde{F}^{*}\left(\tau_{\nu}, \gamma^{*}\left(T_{\nu}(z)\right)\right) \gamma^{* \prime}\left(T_{\nu}(z)\right)^{q}<+\infty .
$$

Furthermore we easily see the following:

$$
\begin{array}{ll}
\lim _{\nu \rightarrow \infty}\left(a_{i}\left(\tau_{\nu}\right)-d_{\nu}\right)=a_{i}\left(\tau_{0}\right)-1 \neq 0 & (i=1,2, \cdots, u), \\
\lim _{\nu \rightarrow \infty}\left(b_{j}^{*}\left(\tau_{\nu}\right)-d_{\nu}\right)=b_{j}^{*}\left(\tau_{0}\right)-1 \neq \infty & (j=1,2, \cdots, v)
\end{array}
$$

and

$$
\lim _{\nu \rightarrow \infty} T_{\nu}^{\prime}(z)^{q}=\lim _{\nu \rightarrow \infty} d_{\nu}^{q}\left(1-d_{\nu}\right)^{q} /\left(z-d_{\nu}\right)^{2 q}=0 .
$$

Therefore we have $\lim _{\nu \rightarrow \infty} f_{2}\left(\tau_{\nu}, z\right)=0$ for $z \in \Omega^{\prime}\left(\tilde{G}_{r}\left(\tau_{0}\right)\right)$.

Next we will show that $\lim _{\nu \rightarrow \infty} f_{1}\left(\tau_{\nu}, z\right)=f\left(\tau_{0}, z\right)$. We decompose the group $G_{1 r}\left(\tau_{\nu}\right)$ into the following two sets: $\hat{G}_{r}\left(\tau_{\nu}\right)$ (see p. 24) and $G_{1 r}\left(\tau_{\nu}\right) / \hat{G}_{r}\left(\tau_{\nu}\right)$ (we denote it by $G_{r}^{\prime}\left(\tau_{\nu}\right)$ ). We will show that

$$
\lim _{\nu \rightarrow \infty} \sum_{\gamma \in G_{r}^{\prime}\left(\tau_{\nu}\right)} F\left(\tau_{\nu}, \gamma(z)\right) \gamma^{\prime}(z)^{q}=0
$$

Elements of $G_{r}^{\prime}\left(\tau_{\nu}\right)$ are classified into the following $4 g_{r} g_{l}$ types:

(i ) $\gamma=W A_{r, h}\left(\tau_{\nu}, z\right) A_{l, k}\left(\tau_{\nu}, z\right) W_{2}$,

(ii) $\gamma=W_{1} A_{r, h}\left(\tau_{\nu}, z\right) A_{l, k}\left(\tau_{\nu}, z\right)^{-1} W_{2}$,

(iii) $\gamma=W_{1} A_{r, h}\left(\tau_{\nu}, z\right)^{-1} A_{l, k}\left(\tau_{\nu}, z\right) W_{2}$ and

(iv) $\gamma=W_{1} A_{r, h}\left(\tau_{\nu}, z\right)^{-1} A_{l, k}\left(\tau_{\nu}, z\right)^{-1} W_{2}$, 
where $W_{1} \in \hat{G}_{r}\left(\tau_{\nu}\right), W_{1} \neq V_{1} A_{r, h}\left(\tau_{\nu}, z\right)^{-n}(n>0)$ with $V_{1} \in \hat{G}_{r}\left(\tau_{\nu}\right)$ for (i) and (ii); $W_{1} \in \hat{G}_{r}\left(\tau_{\nu}\right), W_{1} \neq V_{1} A_{r, h}\left(\tau_{\nu}, z\right)^{n}(n>0), V_{1} \in \hat{G}_{r}\left(\tau_{\nu}\right)$ for (iii) and (iv); $W_{2}$ $\in \tilde{G}_{r}\left(\tau_{\nu}\right), W_{2} \neq A_{l, k}\left(\tau_{\nu}, z\right)^{-n} V_{2}(n>0)$ with $V_{2} \in \tilde{G}_{r}\left(\tau_{\nu}\right)$ for (i) and (iii); $W_{2} \in$ $\tilde{G}_{r}\left(\tau_{\nu}\right), W_{2} \neq A_{l, k}\left(\tau_{\nu}, z\right)^{n} V_{2}(n>0)$ with $V_{2} \in \tilde{G}_{r}\left(\tau_{\nu}\right)$ for (ii) and (iv).

Here we will only consider the case (i) for fixed $h$ and $k$. We can similarly treat the other cases. We denote by $G_{h, k}^{\prime}\left(\tau_{\nu}\right)$ the set of all elements of the type (i) for fixed $h$ and $k$, and denote by $\hat{G}_{h, k}^{\prime}\left(\tau_{\nu}\right)$ the set of all elements $W_{1}=A_{1} A_{2} \cdots A_{n}$ so that each $A_{i}\left(\tau_{\nu}, z\right)(i=1,2, \cdots, n)$ is one of $A_{r, j}\left(\tau_{\nu}, z\right)\left(j=1,2, \cdots, g_{r}\right)$ with $A_{n}\left(\tau_{\nu}, z\right) \neq A_{r, h}\left(\tau_{\nu}, z\right)^{-1}$.

Set

$$
\hat{F}_{h, k}\left(\tau_{\nu}, z\right)=\sum_{\gamma \in \hat{\sigma}_{h, k}^{\prime}\left(\tau_{\nu}\right)} F\left(\tau_{\nu}, \gamma(z)\right) \gamma^{\prime}(z)^{q}
$$

This series converges absolutely and uniformly on any compact set in $\Omega^{\prime}\left(\tilde{G}_{r}\left(\tau_{0}\right)\right)$.

We have the following decomposition into mutually disjoint sets.

$$
G_{h, k}^{\prime}\left(\tau_{\nu}\right)=\hat{G}_{h, k}^{\prime}\left(\tau_{\nu}\right) \hat{\gamma}_{1} \cup \hat{G}_{h, k}^{\prime}\left(\tau_{\nu}\right) \hat{\gamma}_{2} \cup \cdots ;
$$

each $\hat{\gamma}_{n}$ is of form $A_{r, h}\left(\tau_{\nu}, z\right) A_{l, k}\left(\tau_{\nu}, z\right) V_{2}$. With respect to the fundamental domain $\omega_{r}\left(\tau_{\nu}\right)$ and the defining curves $C_{l, k}^{\prime}\left(\tau_{\nu}\right)$ introduced in the proof of Proposition 5, we have

$$
\hat{\gamma}_{n}\left(\omega_{r}\left(\tau_{\nu}\right)\right)=A_{r, h}\left(\tau_{\nu}, A_{l, k}\left(\tau_{\nu}, B_{2}\left(\omega_{r}\left(\tau_{\nu}\right)\right)\right)\right) \subset\left[A_{r, h}\left(\tau_{\nu}, C_{l, k}^{\prime}\left(\tau_{\nu}\right)\right)\right],
$$

where $[A(\cdot)]$ means the interior to $A(\cdot)$.

Set

$$
f_{h, k}\left(\tau_{\nu}, z\right)=\sum_{r \in G_{h, k}^{\prime}\left(\tau_{\nu}\right)} F\left(\tau_{\nu}, \gamma(z)\right) \gamma^{\prime}(z)^{q}
$$

Then

$$
f_{h, k}\left(\tau_{\nu}, z\right)=\sum_{n=1}^{\infty} \hat{F}_{h, k}\left(\tau_{\nu}, \hat{\gamma}_{n}(z)\right) \hat{\gamma}_{n}^{\prime}(z)^{q} .
$$

It is easily seen that $\hat{F}_{h, k}\left(\tau_{\nu}, z\right)$ is holomorphic in $\left[A_{r, h}\left(\tau_{\nu}, C_{l, k}^{\prime}\left(\tau_{\nu}\right)\right)\right]$. Thus by using the same method as in p. 93 , we see that

$$
\left|\hat{F}_{h, k}\left(\tau_{\nu}, z\right)\right| \lambda\left(\tau_{\nu}, z\right)^{2-q} \leqq M
$$

in $\left[A_{r, h}\left(\tau_{\nu}, C_{l, k}^{\prime}\left(\tau_{\nu}\right)\right)\right]$ for some constant $M$ which does not depend on $\nu$. Thus by the same way as before, we have

$$
\iint_{\omega_{r}\left(\tau_{\nu}\right)}\left|f_{h, k}\left(\tau_{\nu}, z\right)\right| \lambda\left(\tau_{\nu}, z\right)^{2-q} d x d y \leqq M \text { Area }\left[A_{r, h}\left(\tau_{\nu}, C_{l, k}^{\prime}\left(\tau_{\nu}\right)\right)\right]
$$


Since Area $\left[A_{r, h}\left(\tau_{\nu}, C_{l, k}^{\prime}\left(\tau_{\nu}\right)\right)\right]$ tend to 0 as $\nu \rightarrow \infty$, we have

$$
\lim _{\nu \rightarrow \infty} f_{h, k}\left(\tau_{\nu}, z\right)=0 \quad \text { for } z \in \Omega^{\prime}\left(\tilde{G}_{r}\left(\tau_{0}\right)\right) \text {. }
$$

Hence we conclude

$$
\lim _{\nu \rightarrow \infty} f_{1}\left(\tau_{\nu}, z\right)=f\left(\tau_{0}, z\right) .
$$

Our proof is now complete.

6-3. Proposition 11. Under the same assumption as in Proposition 5 , if $F\left(\tau_{\nu}, z\right)$ is one of the functions $\Psi_{k}\left(\tau_{\nu}, z\right) \in L_{s}\left(\tau_{\nu}\right)$ such that $\Psi_{k}\left(\tau_{0}, z\right) \in L_{s}\left(\tau_{0}\right)$ with $s \in W_{r}(L)$, then (13) and (17) are satisfied, where $f$ and $f^{*}$ are defined from $F$ in $\S 4-2$.

Proof. It suffices to consider the following function:

$$
F\left(\tau_{\nu}, z\right)=1 /\left(\prod_{i=1}^{u}\left(z-a_{i}\left(\tau_{\nu}\right)\right)^{m_{i}} \prod_{j=1}^{v}\left(z-b_{j}\left(\tau_{\nu}\right)\right)^{n_{j}}\right),
$$

with $m_{1}=q, \quad m_{i} \geqq 0(i=2,3, \cdots, u), \quad n_{j} \geqq 0(j=1,2, \cdots, v), \quad \sum_{i=1}^{u} m_{i}+$ $\sum_{j=1}^{v} n_{j}=2 q$ and $a_{1}\left(\tau_{\nu}\right) \in \hat{P}_{r 3}\left(\tau_{\nu}\right), a_{i}\left(\tau_{\nu}\right) \in \hat{P}_{r}\left(\tau_{\nu}\right)(i=2,3, \cdots, u), b_{j}\left(\tau_{\nu}\right) \in \hat{P}_{l}\left(\tau_{\nu}\right)$ $(j=1,2, \cdots, v)$, or $m_{i} \geqq 0(i=1,2, \cdots, u), n_{1}=q, n_{j}=0(j=2,3, \cdots, v)$, $\sum_{i=1}^{u} m_{i}+\sum_{j=1}^{v} n_{j}=2 q$, and $a_{i}\left(\tau_{\nu}\right) \in \hat{P}_{r}\left(\tau_{\nu}\right), b_{1}\left(\tau_{\nu}\right) \in \hat{P}_{l 3}\left(\tau_{\nu}\right)$.

This proposition is proved by modifying the proofs of Propositions 5, 6, 7, and 8, when $n_{1}+\cdots+n_{v} \leqq q-1$. Furthermore we can prove the proposition by the same methods as the proofs of Propositions 5 and 10 , when $n_{1}+\cdots+n_{v}=q$.

6-4. Proposition 12. Under the same assumption as in Proposition 5 , let $F\left(\tau_{\nu}, z\right)$ be one of the functions $\Psi_{s, \sigma}\left(\tau_{\nu}, z\right)$ with $s \in W_{r}(J)$. If $F\left(\tau_{0}, z\right)$ is one of the following functions (i) $F\left(\tau_{0}, z\right)=\Psi_{s, \sigma}\left(\tau_{0}, z\right)$ with $\sigma \in \mathscr{S}_{s}^{I, L}$ and $s \in$ $W_{r}(L)$, (ii) $F\left(\tau_{0}, z\right) \in M_{s}\left(\tau_{0}\right)$ with $s \in W_{r}(L)$, and (iii) $F\left(\tau_{0}, z\right) \in L_{s}\left(\tau_{0}\right)$ with $s \in$ $W_{r}(L)$, then (13) and (17) are satisfied, where $f$ and $f^{*}$ are defined from $F$ in $\S 4-2$.

Proof. For the cases (i) and (ii), the proposition is similarly proved by modifying the proofs of Propositions 5, 6, 7, and 8. For the case (iii), we can prove the proposition by similar methods to the proofs of Propositions 5 and 10.

6-5. For the sake of convenience, we summarize from Proposition 5 through Proposition 12 in the following form. 
Proposition 13. Let $I \subset\{1,2, \cdots, g\}$ and $J \subset\{1,2, \cdots, 2 g-3\}$. Let $F(\tau, z)$ be one of the functions $\Phi_{s, h}(\tau, z), \Theta_{s, \pi}(\tau, z), \Psi_{k}(\tau, z)$ with $k \in J_{s}$ and $\Psi_{s, \sigma}(\tau, z)$ defined on $\sum_{t=0}^{2 g-3} \widetilde{F}_{t} \Im_{g}^{K, L}(\Sigma)$, and let $f(\tau, z)$ be defined from $F$ as in $\S 4-2$.

(i) Suppose $|I|<g, k \in\{1,2, \cdots, g\} \backslash I$ and $\left\{\tau_{\nu}\right\}$ is a sequence of points in $\sigma^{I, J} \widetilde{S}_{g}(\Sigma)$ such that $\lim _{\nu \rightarrow \infty} \tau_{\nu}=\tau_{0} \in \delta^{K, J} \widetilde{S}_{g}(\Sigma)$ with $K=I \cup\{k\}$. Then

$$
\lim _{\nu \rightarrow \infty} f\left(\tau_{\nu}, z\right)=f\left(\tau_{0}, z\right) \quad \text { for } z \in \Omega^{\prime}\left(\tilde{G}_{t}\left(\tau_{0}\right)\right), t=0,1, \cdots, 2 g-3 .
$$

(ii) Suppose $|J|<2 g-3, l \in\{1,2, \cdots, 2 g-3\} \mid J$ and $\left\{\tau_{\nu}\right\}$ is a sequence of points in $\delta^{I, J} \widetilde{S}_{g}(\Sigma)$ such that $\lim _{\nu \rightarrow \infty} \tau_{\nu}=\tau_{0} \in \delta^{I, L} \widetilde{S}_{g}(\Sigma)$ with $L=J \cup\{l\}$. Then

$$
\lim _{\nu \rightarrow \infty} f\left(\tau_{\nu}, z\right)=f\left(\tau_{0}, z\right) \quad \text { for } z \in \Omega^{\prime}\left(\tilde{G}_{t}\left(\tau_{0}\right)\right), t=0,1, \cdots, 2 g-3 .
$$

From Proposition 13, we obtain the following proposition by modifying the proof in Bers [2].

Proposition 14. The functions $\phi_{s, h}(\tau, z), \theta_{s, \pi}(\tau, z), \psi_{k}(\tau, z)$ are holomorphic functions of $(\tau, z) \in \sum_{t=0}^{2 g-3} \widetilde{\mho}_{t} \hat{\Theta}_{g}^{*}(\Sigma)$. The functions $\psi_{s, \sigma}(\tau, z)$ with $\sigma \epsilon$ $\mathscr{S}_{s}^{K, L}(\Sigma)$, are holomorphic functions of $(\tau, z) \in \sum_{t=0}^{2 g-3} \mathfrak{\mho}_{t} \mathfrak{S}_{g}^{K, L}(\Sigma)$.

\section{§7. Linear independence}

Let $\tau \in \delta^{I, J} \widetilde{S}_{g}(\Sigma)$. We recall that $g_{s}=g_{s}(\tau), k_{s}=k_{s}(\tau)$ and $l_{s}=l_{s}(\tau)$ mean the genus of $\tilde{S}_{s}(\tau)$, the half of the number of the distinguished points of the first kind on $\tilde{S}_{s}(\tau)$ and the number of the distinguished points of the second kind on $\tilde{S}_{s}(\tau)$, respectively.

7-1. Proposition 15. For each $s=0,1, \cdots, 2 q-3$, there exist $(2 q-1)\left(g_{s}+k_{s}-1\right)+n_{s} q$ linearly independent $q$-forms $\phi_{s, h}(\tau, z)(h=1,2, \cdots$, $\left.g_{s}+k_{s}\right), \theta_{s, \pi_{i}}(\tau, z)\left(i=1,2, \cdots, e_{s}\right)$, and $\psi_{j}(\tau, z)$ with $j \in J_{s}\left(\left|J_{s}\right|=n_{s}\right)$ for every $\tau \in \delta^{I, J \Im_{g}}(\Sigma)$, provided $g_{s} \leqq 1$, where $e_{s}=\left\{2\left(g_{s}+k_{s}\right)+n_{s}-2\right\} q-$ $\left\{2\left(g_{s}+k_{s}\right)+n_{s}\right\}+1$ (see p. 83).

We can prove it by a method similar to that of proof in [2].

As in $\S 3-4$, we consider the projections $\hat{\phi}_{s, h}, \hat{\theta}_{s, \pi i}, \hat{\psi}_{j}$ and $\hat{\psi}_{s, \sigma}$ on $S(\tau)$ of the functions $\phi_{s, h}, \theta_{s, \pi_{i}}, \psi_{j}$ and $\psi_{s, \sigma}$, respectively. Suppose $\tilde{S}_{s}(\tau)=\tilde{S}_{t}(\tau)$ and $\tilde{G}_{t}(\tau)=T \tilde{G}_{s}(\tau) T^{-1}$ with $T \in$ Möb. Then we may regard $\hat{\phi}_{s, h}(\tau, z)=$ $\hat{\phi}_{t, h}(\tau, z), \hat{\theta}_{s, \pi_{i}}(\tau, z)=\hat{\theta}_{t, \pi_{i}}(\tau, z), \ldots$. Thus we have the following from Proposition 15 . 
Corollary. Let $\tau \in \delta^{I, J \widetilde{S}_{g}}(\Sigma)$ with $J=\left\{j_{1}, \cdots, j_{m}\right\}$. For each $k=0$, $1, \cdots, m$, assume $g_{j_{k}} \leqq 1$, where $g_{j_{0}}=g_{0}$. Then $(2 q-1)(q-1)$ differentials on $S(\tau), \hat{\phi}_{s, h}(\tau, z)\left(s=0, j_{1}, \cdots, j_{m} ; h=1,2, \cdots, g_{s}+k_{s}\right), \theta_{s, \pi i}(\tau, z)(s=0$, $\left.j_{1}, \cdots, j_{m} ; i=1,2, \cdots, e_{s}(\tau)\right)$, and $\psi_{j}(\tau, z)\left(j=j_{1}, \cdots, j_{m}\right)$ are linearly independent.

7-2. Let $J=\left\{j_{1}, \cdots, j_{m}\right\} . \quad$ For $\tau_{0} \in \delta^{J} \widetilde{S}_{g}^{I}(\Sigma)$, let $\tilde{G}_{s}\left(\tau_{0}\right)=\left\langle A_{s, 1}\left(\tau_{0}, z\right), \cdots\right.$, $\left.A_{s, g_{s}}\left(\tau_{0}, z\right)\right\rangle$ which represent Riemann surfaces $\tilde{S}_{s}\left(\tau_{0}\right)\left(s=0, j_{1}, \cdots, j_{m}\right)$. Let $J^{\prime}=\left\{j_{1}^{\prime}, \cdots, j_{m^{\prime}}^{\prime}\right\} \subset J$. For $\tau \in \delta^{J^{\prime}} \mathfrak{S}_{g}^{I}(\Sigma)$, we set

$\hat{G}_{s}(\tau)=\left\langle A_{s, 1}(\tau, z), \cdots, A_{s, g_{s}}(\tau, z)\right\rangle\left(s=0, j_{1}, \cdots, j_{m}\right)$. We recall the notations $P_{s}(\tau)$ (see p. 82) and $\hat{P}_{s}(\tau)$ (see p. 86). Let $\gamma_{j_{s}}\left(\tau_{0}\right)=\gamma\left(1, i_{1}, \cdots, i_{\mu(s)}\right)\left(\tau_{0}\right)$ $(s=1,2, \cdots, m)$. Let $\gamma_{j_{t}}\left(\tau_{0}\right)=\gamma\left(1, i_{1}, \cdots, i_{\mu(s)}, i_{\mu(s)+1}, \cdots, i_{\mu(t)}\right)\left(\tau_{0}\right)$ be the nodes joining $\tilde{S}_{j_{s}}\left(\tau_{0}\right)$ and $\tilde{S}_{j_{t}}\left(\tau_{0}\right)$. Assume that $j_{t} \notin J^{\prime}$. Then we note that $\tilde{S}_{j_{s}}(\tau)$ $=\tilde{S}_{j_{t}}(\tau)$ for $\tau \in \delta^{J^{\prime} S_{g}^{*}}(\Sigma)$. In $\S 5-2$ in [4], [ $\left.\sigma_{j}\right]$ represents the part of $S$ divided by the loops $\gamma_{j}$, with $j^{\prime} \in J^{\prime}$ which contains the cell $\sigma_{j}$.

We denote by $\bar{i}_{\mu}$ the number $1-i_{\mu}$. We define $p^{s}(\tau), q^{s}(\tau), p^{t}(\tau)$ and $q^{t}(\tau)$ as follows:

$$
p^{s}(\tau)= \begin{cases}p_{1}(\tau) & \text { if } \sigma_{0} \in\left[\sigma_{j_{s}}\right] \\ p^{-}\left(1, i_{1}, \cdots, i_{\nu}\right)(\tau) & \text { if } \sigma_{0} \notin\left[\sigma_{j_{s}}\right]\end{cases}
$$

where $p^{-}\left(1, i_{1}, \cdots, i_{\nu}\right)(\tau)$ is the left distinguished point whose projection lies on $\tilde{S}_{j_{s}}(\tau)$;

$$
q^{s}(\tau)=\left\{\begin{array}{ll}
p\left(1, i_{1}, \cdots, i_{\mu(s)}, \bar{i}_{\mu(s)+1}, 0, \cdots, 0\right)(\tau)=p_{l}(\tau) & \text { if } \sigma_{l} \in\left[\sigma_{j_{s}}\right] \\
p^{+}\left(1, i_{1}, \cdots, i_{\mu(s)}, \bar{i}_{\mu(s)+1}, 0, \cdots, 0\right)(\tau) & \text { if } \sigma_{l} \notin\left[\sigma_{j_{s}}\right]
\end{array},\right.
$$

where $\sigma_{l}$ is the terminal cell and $p^{+}\left(1, i_{1}, \cdots, i_{\mu(s)}, \bar{i}_{\mu(s)+1}, 0, \cdots, 0\right)(\tau)$ is the right distinguished point whose projection lies on $\tilde{S}_{j_{s}}(\tau)$;

$$
p^{t}(\tau)= \begin{cases}p\left(1, i_{1}, \cdots, i_{\mu(t)}, 0, \cdots, 0\right)(\tau)=p_{k}(\tau) & \text { if } \sigma_{k} \in\left[\sigma_{j_{t}}\right], \\ p^{+}\left(1, i_{1}, \cdots, i_{\mu(t)}, 0, \cdots, 0\right)(\tau) & \text { if } \sigma_{k} \notin\left[\sigma_{j_{t}}\right]\end{cases}
$$

where $\sigma_{k}$ is the terminal cell and $p^{+}\left(1, i_{1}, \cdots, i_{\mu(t)}, 0, \cdots, 0\right)(\tau)$ is the right distinguished point whose projection lies on $\tilde{S}_{j_{t}}(\tau)$;

$$
q^{t}(\tau)=\left\{\begin{array}{ll}
p\left(1, i_{1}, \cdots, i_{\mu(t)}, 1,0, \cdots, 0\right)(\tau)=p_{h}(\tau) & \text { if } \sigma_{h} \in\left[\sigma_{j_{s}}\right] \\
p^{+}\left(1, i_{1}, \cdots, i_{\mu(t)}, 1,0, \cdots, 0\right) & \text { if } \sigma_{h} \notin\left[\sigma_{j_{t}}\right]
\end{array} .\right.
$$

where $\sigma_{h}$ is the terminal cell and $p^{+}\left(1, i_{1}, \cdots, i_{\mu(t)}, 1,0, \cdots, 0\right)(\tau)$ is the right distinguished point whose projection lies on $\tilde{S}_{j_{t}}(\tau)$. 
7-3. Let $\tau_{0} \in \delta^{I, J} \widetilde{S}_{g}(\Sigma)$ with $J=\left\{j_{1}, \cdots, j_{m}\right\}$. Then there exist $d=$ $(2 q-1)(g-1)$ numbers of regular $q$-differentials $\hat{f}_{s, i}(\tau, \hat{z})\left(s=0, j_{1}, j_{2}, \cdots\right.$, $\left.j_{m} ; i=1,2, \cdots, d_{s}-n_{s}\right)$ and $\hat{f}_{j_{t}}(\tau, \hat{z})(t=1,2, \cdots, m)$ for $\hat{z} \in S(\tau)$ and $\tau \in$ $\mathfrak{S}_{g}^{I, J}(\Sigma)$ satisfying the following properties (i) and (ii), where $d_{s}=d_{s}(\tau)=$ $(2 q-1)\left(g_{s}+k_{s}-1\right)+q n_{s}:$

(i) For $\tau \in \mathbb{S}_{g}^{I, J}(\Sigma)$, let $f_{s, i}(\tau, z)$ be linear combination of functions $\phi_{s, h}(\tau, z)\left(h=1,2, \cdots, g_{s}+k_{s}\right), \psi_{s, \sigma}(\tau, z)$ with $\sigma \in \mathscr{S}_{s}^{I, J}$ and

$$
\theta_{s, \pi}(\tau, z)=\sum_{r \in \tilde{\sigma}_{s}(\tau)} \Theta_{s, \pi}(\tau, \gamma(z)) \gamma^{\prime}(z)^{q},
$$

where

$$
\Theta_{s, \pi}(\tau, z)=1 /\left(\prod_{i=1}^{u}\left(\left(z-a_{i}(\tau)\right)^{m_{i}} \prod_{j=1}^{v}\left(z-b_{j}(\tau)\right)^{n_{j}}\right)\right.
$$

with $\sum_{\imath=1}^{u} m_{i}+\sum_{j=1}^{v} n_{j}=2 q, 1 \leqq m_{i} \leqq q-1(i=1,2, \cdots, u), 0 \leqq \sum_{j=1}^{v} n_{j}$ $\leqq q-1, a_{i}(\tau) \in \hat{P}_{s}(\tau)$ and $b_{j}(\tau) \in P_{s}(\tau) \backslash \hat{P}_{s}(\tau)$, where $a_{i}(\tau)$ and $b_{j}(\tau)$ are selected as in $\S 3-2$, pp. 87-88. Then $\hat{f}_{s, i}(\tau, \hat{z})$ are the projections of $f_{s, i}(\tau, z)$.

(ii) Let $\gamma_{j_{t}}\left(\tau_{0}\right)$ be the node joining $\tilde{S}_{j_{s}}\left(\tau_{0}\right)$ and $\tilde{S}_{j_{t}}\left(\tau_{0}\right)$. For $\tau \in \delta^{I, J^{\prime} \widetilde{S}_{g}}(\Sigma)$, set

$$
f_{j_{t}}(\tau, z)=\sum_{\tilde{\sigma}_{j_{s}}(\tau)} \frac{\gamma^{\prime}(z)^{q}}{\left(\gamma(z)-p^{s}(\tau)\right)^{q-1}\left(\gamma(z)-q^{s}(\tau)\right)\left(\gamma(z)-p^{t}(\tau)\right)^{q-1}\left(\gamma(z)-q^{t}(\tau)\right)}
$$

if $j_{t} \notin J^{\prime}$, and

$$
f_{j_{t}}(\tau, z)=\sum_{\hat{\sigma}_{j_{s}}(\tau)} \frac{\gamma^{\prime}(z)^{q}}{\left(\gamma(z)-p^{s}(\tau)\right)^{q-1}\left(\gamma(z)-q^{s}(\tau)\right)\left(\gamma(z)-p_{j_{t}}^{+}(\tau)\right)^{q}}
$$

if $j_{t} \in J^{\prime}$. Then $\hat{f}_{j_{t}}(\tau, \hat{z})$ are the projections of $f_{j_{t}}(\tau, z)$.

Proposition 16. $\quad d=(2 q-1)(g-1)$ numbers of $q$-differentials $\hat{f}_{s, i}\left(\tau_{0}, \hat{z}\right)$ and $\hat{f}_{j_{t}}\left(\tau_{0}, \hat{z}\right)$ are linearly independent on $S\left(\tau_{0}\right)$.

A proof of the proposition is performed similarly to that in Bers [2]. We define

$$
f_{j_{t}}\left(\tau_{0}, z\right)=\lim _{\nu \rightarrow \infty} f_{j_{t}}\left(\tau_{\nu}, z\right), \quad \text { for } z \in \Omega^{\prime}\left(\tilde{G}_{j s}\left(\tau_{0}\right)\right)
$$

which is equal to

$$
\sum_{\tilde{G}_{j_{s}\left(\tau_{0}\right)}} \gamma^{\prime}(z)^{q} /\left(\left(\gamma(z)-p^{s}\left(\tau_{0}\right)\right)^{q-1}\left(\gamma(z)-q^{s}\left(\tau_{0}\right)\right)(\gamma(z)-1)^{q}\right) .
$$

Next for $\tau_{\nu} \in \delta^{I, J^{\prime} \subseteq_{g}}(\Sigma)$ with $J^{\prime} \subseteq J, j_{t} \notin J^{\prime}$, we set $f_{j_{t}}^{*}\left(\tau_{\nu}, w\right)=f_{j_{t}}\left(\tau_{\nu}\right.$, 
$\left.T_{\nu}^{-1}(w)\right) T_{\nu}^{-1^{\prime}}(w)^{q}, \quad w \in \Omega^{\prime}\left(\tilde{G}_{j_{t}}\left(\tau_{\nu}\right)\right)$, where $T_{\nu} \in$ Möb with $\tilde{G}_{j_{t}}\left(\tau_{\nu}\right)=T_{\nu} \tilde{G}_{j_{s}}\left(\tau_{\nu}\right) T_{\nu}^{-1}$. Then we define

$$
f_{J t}^{*}\left(\tau_{0}, w\right)=\lim _{\nu \rightarrow \infty} f_{J t}^{*}\left(\tau_{\nu}, w\right)
$$

which is equal to

$$
\begin{aligned}
\sum_{\tilde{\sigma}_{j_{t}\left(\tau_{0}\right)}} & \frac{\gamma^{* \prime}(w)^{q}}{\left(\gamma^{*}(w)-p^{t}\left(\tau_{0}\right)^{*}\right)^{q-1}\left(\gamma^{*}(w)-q^{t}\left(\tau_{0}\right)^{*}\right)\left(\gamma^{*}(w)-1\right)^{q}} \\
& \times \frac{(-1)^{q}\left(p^{t}\left(\tau_{0}\right)^{*}-1\right)^{q-1}\left(q^{t}\left(\tau_{0}\right)^{*}-1\right)}{\left(p^{s}\left(\tau_{0}\right)-1\right)^{q-1}\left(q^{s}\left(\tau_{0}\right)-1\right)}
\end{aligned}
$$

for $w \in \Omega^{\prime}\left(\tilde{G}_{j_{t}}\left(\tau_{0}\right)\right)$, where $p^{t}\left(\tau_{0}\right)^{*}=\lim _{\nu \rightarrow \infty} T_{\nu}\left(p^{t}\left(\tau_{\nu}\right)\right)$ and $q^{t}\left(\tau_{0}\right)^{*}=\lim _{\nu \rightarrow \infty} T_{\nu}\left(q^{t}\left(\tau_{\nu}\right)\right)$.

Remark. From Lemma 1 , each $\hat{f}_{s, i}\left(\tau_{0}, \hat{z}\right)\left(s=0, j_{1}, \cdots, j_{m} ; i=1,2, \cdots\right.$, $\left.d_{s}\left(\tau_{0}\right)-n_{s}\left(\tau_{0}\right)\right)$ is identically zero on $S\left(\tau_{0}\right) \backslash S_{s}\left(\tau_{0}\right)$.

7-4. Proposition 17. The q-differentials in Proposition 16 are linearly independent on $S(\tau)$ for sufficiently close to $\tau_{0}$.

Proof. If $\tau \in \delta^{I^{\prime}, J} \widetilde{S}_{g}(\Sigma)$ with $I^{\prime} \subset I$, the proposition is proved by a similar method in Bers [2]. Thus we will show it in the case of $\tau \in \delta^{I, J^{\prime}} \subseteq_{g}(\Sigma)$ with $J^{\prime} \subset J$.

It suffices to show the proposition in the case of $J^{\prime}=J \backslash\{l\}$, since we can show it similarly in the general case. For simplicity, we assume that $J=\{l\}$ and $J^{\prime}=\phi$. Two parts of $S\left(\tau_{0}\right)$ joined by the node $\gamma_{l}\left(\tau_{0}\right)$ are $\tilde{S}_{0}\left(\tau_{0}\right)$ and $\tilde{S}_{l}\left(\tau_{0}\right)$. We write $g_{0}, g_{1}, d_{0}$ and $d_{1}$ instead of $g_{0}\left(\tau_{0}\right), g_{l}\left(\tau_{0}\right), d_{0}\left(\tau_{0}\right)$ and $d_{l}\left(\tau_{0}\right)$, respectively.

For simplicity, we introduce the following notations

$$
\hat{f}_{1}(\tau, \hat{z}), \quad \hat{f}_{2}(\tau, \hat{z}), \cdots, \hat{f}_{d}(\tau, \hat{z}), \quad \tau \in \widetilde{S}_{g}^{I,\{l\}}(\Sigma)
$$

as follows:

(i) For $\tau \in \mathfrak{S}_{g}^{I}(\Sigma), f_{1}(\tau, z), \cdots, f_{d_{0}-1}(\tau, z) ; f_{d_{0}+1}(\tau, z), \cdots, f_{d_{0}+d_{1}}(\tau, z)$ are functions as in p. 58. Then $\hat{f}_{h}(\tau, \hat{z})\left(h=1,2, \cdots, d_{0}-1, d_{0}+1, \cdots, d_{0}+d_{1}\right)$ are the projections of $f_{h}(\tau, z)$ onto $S(\tau)$.

(ii) For $\tau \in \mathfrak{S}_{g}^{I}(\Sigma), f_{d_{0}}(\tau, z)$ is a function as in p. 59. Then $\hat{f}_{a_{0}}(\tau, \hat{z})$ is the projection of $f_{d_{0}}(\tau, z)$ onto $S(\tau)$.

Remember that

$$
f_{1}\left(\tau_{0}, z\right), \cdots, f_{d_{0}}\left(\tau_{0}, z\right), \quad z \in \Omega^{\prime}\left(\tilde{G}_{0}\left(\tau_{0}\right)\right)
$$

and 


$$
f_{d_{0}}\left(\tau_{0}, z\right), \cdots, f_{d_{0}+d_{1}}\left(\tau_{0}, z\right), \quad z \in \Omega^{\prime}\left(\tilde{G}_{l}\left(\tau_{0}\right)\right)
$$

are linearly independent on $\Omega^{\prime}\left(\tilde{G}_{0}\left(\tau_{0}\right)\right)$ and $\Omega^{\prime}\left(\tilde{G}_{l}\left(\tau_{0}\right)\right)$, respectively, and that $f_{i}\left(\tau_{0}, z\right)=0$ on $\Omega^{\prime}\left(\tilde{G}_{l}\left(\tau_{0}\right)\right)$ for $i=1,2, \cdots, d_{0}-1$, and $f_{i}\left(\tau_{0}, z\right)=0$ on $\Omega^{\prime}\left(\tilde{G}_{0}\left(\tau_{0}\right)\right)$ for $i=d_{0}+1, \cdots, d_{0}+d_{1}$.

It is necessary and sufficient for $f_{i}\left(\tau_{0}, z\right), i=1,2, \cdots, n$, being linearly independent on $\Omega^{\prime}\left(\tilde{G}_{0}\left(\tau_{0}\right)\right)$ that for $z_{0} \in \Omega^{\prime}\left(\tilde{G}_{0}\left(\tau_{0}\right)\right)$ and for sufficiently small $\varepsilon$, the Gram determinant is positive, namely if we write

$$
\begin{aligned}
& \left(f_{i}\left(\tau_{0}, \cdot\right), f_{j}\left(\tau_{0}, \cdot\right)\right)_{z_{0}} \\
& \quad=\iint_{\left|z-z_{0}\right|<\varepsilon} f_{i}\left(\tau_{0}, z\right) \overline{f_{j}\left(\tau_{0}, z\right) \lambda\left(\tau_{0}, z\right)^{2-2 q} d x d y,}
\end{aligned}
$$

then

$$
\operatorname{det}\left(\left(f_{i}\left(\tau_{0}, \cdot\right), f_{j}\left(\tau_{0}, \cdot\right)\right)_{z_{0}}\right)>0 \quad(i, j=1,2, \cdots, n),
$$

where $\lambda\left(\tau_{0}, z\right)$ is the Poincaré metric on $\Omega^{\prime}\left(\tilde{G}_{0}\left(\tau_{0}\right)\right)$. Thus we have

$$
\operatorname{det}\left(\left(f_{i}\left(\tau_{0}, \cdot\right), f_{j}\left(\tau_{0}, \cdot\right)\right)_{z_{0}}\right)>0 \quad\left(i, j=1,2, \cdots, d_{0}\right)
$$

and

$$
\operatorname{det}\left(\left(f_{i}\left(\tau_{0}, \cdot\right), f_{j}\left(\tau_{0}, \cdot\right)\right)_{z_{0}}\right)>0 \quad\left(i, j=1,2, \cdots, d_{0}-1\right) .
$$

Similarly we have that for $z_{1} \in \Omega^{\prime}\left(\tilde{G}_{l}\left(\tau_{0}\right)\right)$,

$$
\operatorname{det}\left(\left(f_{i}\left(\tau_{0}, \cdot\right), f_{j}\left(\tau_{0}, \cdot\right)\right)_{z_{1}}\right)>0 \quad\left(i, j=d_{0}, \cdots, d_{0}+d_{1}\right)
$$

and

$$
\operatorname{det}\left(\left(f_{i}\left(\tau_{0}, \cdot\right), f_{j}\left(\tau_{0}, \cdot\right)\right)_{z_{1}}\right)>0 \quad\left(i, \mathrm{j}=d_{0}+1, \cdots, d_{0}+d_{1}\right) .
$$

Let $\tau \in \mathbb{S}_{g}^{I,\{l]}(\Sigma)$ be sufficiently close to $\tau_{0}$. We set

$$
a_{i j}(\tau)=\left(f_{i}(\tau, \cdot), f_{j}(\tau, \cdot)\right)_{z_{0}} \quad\left(i, j=1,2, \cdots, d_{0}+d_{1}\right)
$$

for $z_{0} \in \Omega^{\prime}\left(\tilde{G}_{0}(\tau)\right)$ and

$$
b_{i j}(\tau)=\left(f_{i}(\tau, \cdot), f_{j}(\tau, \cdot)\right)_{z_{1}} \quad\left(i, j=1,2, \cdots, d_{0}+d_{1}\right)
$$

for $z_{1} \in \Omega^{\prime}\left(\tilde{G}_{l}(\tau)\right)$. Then if we show that

$$
\operatorname{det}\left(a_{i j}\left(\tau_{0}\right)+b_{i j}\left(\tau_{0}\right)\right)>0 \quad\left(i, j=1,2, \cdots, d_{0}+d_{1}\right)
$$

then we have

$$
\operatorname{det}\left(a_{i j}(\tau)+b_{i j}(\tau)\right)>0 \quad\left(i, j=1,2, \cdots, d_{0}+d_{1}\right)
$$


for $\tau$ near $\tau_{0}$ by using continuity of the functions, and therefore $f_{1}(\tau, z), \cdots$, $f_{d_{0}+d_{1}}(\tau, z)$ are linearly independent on $S(\tau)$ for $\tau$ near $\tau_{0}$.

Set

$$
\begin{array}{ll}
\Lambda_{0}^{\prime}\left(\tau_{0}\right)=\operatorname{det}\left(\left(a_{i j}\left(\tau_{0}\right)\right)\right) & \left(i, j=1,2, \cdots, d_{0}-1\right), \\
\Lambda_{0}\left(\tau_{0}\right)=\operatorname{det}\left(\left(a_{i j}\left(\tau_{0}\right)\right)\right) & \left(i, j=1,2, \cdots, d_{0}\right), \\
\Lambda_{1}^{\prime}\left(\tau_{0}\right)=\operatorname{det}\left(\left(b_{i j}\left(\tau_{0}\right)\right)\right) & \left(i, j=d_{0}+1, \cdots, d_{0}+d_{1}\right)
\end{array}
$$

and

$$
\Lambda_{1}\left(\tau_{0}\right)=\operatorname{det}\left(\left(b_{i j}\left(\tau_{0}\right)\right)\right) \quad\left(i, j=d_{0}, \cdots, d_{0}+d_{1}\right) .
$$

We note that for each $i=1,2, \cdots, d_{0}-1, a_{i j}\left(\tau_{0}\right)=0\left(j=d_{0}+1, \cdots\right.$, $\left.d_{0}+d_{1}\right)$ and $b_{i j}\left(\tau_{0}\right)=0\left(j=1,2, \cdots, d_{0}+d_{1}\right)$; for each $i=d_{0}+1, \cdots, d_{0}$ $+d_{1}, a_{i j}\left(\tau_{0}\right)=0\left(j=1,2, \cdots, d_{0}+d_{1}\right)$ and $b_{i j}\left(\tau_{0}\right)=0\left(j=1,2, \cdots, d_{0}-1\right)$. Then we have that

$$
\operatorname{det}\left(a_{i j}\left(\tau_{0}\right)+b_{i j}\left(\tau_{0}\right)\right)=\Lambda_{0}^{\prime}\left(\tau_{0}\right) \Lambda_{1}\left(\tau_{0}\right)+\Lambda_{0}\left(\tau_{0}\right) \Lambda_{1}^{\prime}\left(\tau_{0}\right) .
$$

From (35) through (38),

$$
\operatorname{det}\left(a_{i j}\left(\tau_{0}\right)+b_{i j}\left(\tau_{0}\right)\right)>0 .
$$

Our proof is now complete.

7-5. We consider the set $Z \subset \widetilde{S}_{g}^{I, J}(\Sigma)$ defined as totality of the $\tau$ for which the $q$-forms are linearly dependent.

Proposition 18. For each $J^{\prime} \subset J$, the set $Z$ is either empty or an analytic subvariety of pure codimension 1 in $\delta^{J^{\prime}} \widetilde{S}_{g}^{I}(\Sigma)$.

This proposition is proved by the method similar to that in [2].

\section{§8. Theorems}

In this section, we will state three theorems. They were obtained in the case of the fiber space of the augmented Schottky space in the sense of Bers by Bers [2]. Our theorems are for the fiber spaces of the augmented Schottky space defined in [5].

8-1. By using the results of the previous sections, Propositions 14, $15,16,17$, and 18, we obtain the following Theorems 1 and 2.

Theorem 1. Let $g \geqq 2$ and $q \geqq 2$ be integers. There exist $d=$ $(2 q-1)(g-1)$ holomorphic functions 


$$
f_{s, 1}(\tau, z), f_{s, 2}(\tau, z), \cdots, f_{s, d}(\tau, z), \quad(\tau, z) \in \sum_{t=0}^{2 g-3} \widetilde{\mho}_{t} \hat{S}_{g}^{*}(\Sigma)
$$

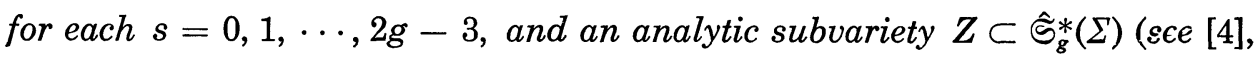
pp. 163-167 for the definition of the augmented Schottky space $\hat{\subseteq}_{g}^{*}(\Sigma)$ ) having the following properties (i)-(v):

(i ) $Z \subset \delta^{J} \Im_{g}^{I}(\Sigma)$ is either empty or of pure codimension 1 in $\delta^{J} \Im_{g}^{I}(\Sigma)$ for $J \subset\{1,2, \cdots, 2 g-3\}$.

(ii) $Z$ avoids all points $\tau$ which lie on any set $\delta^{I, J \Im_{g}}(\Sigma)$ with $g_{t}(\tau) \leqq 1$ for all $t=0,1, \cdots, 2 g-3$, where $g_{t}(\tau)$ represents the genus of the Riemann surface $\tilde{S}_{t}(\tau)$.

(iii) Suppose $\tilde{S}_{s}(\tau)=\tilde{S}_{t}(\tau)$ and $\tilde{G}_{t}(\tau)=T \tilde{G}_{s}(\tau) T^{-1}$ with $T \in$ Möb. Then

$$
f_{s, i}(\tau, z)=f_{t, i}(\tau, T(z)) T^{\prime}(z)^{q} \quad \text { for } z \in \Omega^{\prime}\left(\tilde{G}_{s}(\tau)\right), i=1,2, \cdots, d .
$$

When $\hat{f}_{s, i}(\tau, \hat{z})$ and $\hat{f}_{t, i}(\tau, \hat{z})$ are the projections of $f_{s, i}(\tau, z)$ and $f_{t, i}(\tau, z)$, respectively and $s$ and $t$ have the above relation, we have $\hat{f}_{s, i}(\tau, \hat{z})=\hat{f}_{t, i}(\tau, \hat{z})$. Therefore we define $d$ numbers of $q$-differentials $\hat{f}_{1}(\tau, \hat{z}), \cdots, \hat{f}_{d}(\tau, \hat{z})$ for $\tau \in$ $\hat{\subseteq}_{g}^{*}(\Sigma)$ and $\hat{z} \in S(\tau)$ by setting

$$
\hat{f}_{i}(\tau, \hat{z})=\hat{f}_{s, i}(\tau, \hat{z})
$$

if $z \in \tilde{S}_{s}(\tau), s=0,1, \cdots, 2 g-3$. The fourth property is as follows.

(iv) For each $\tau \in \hat{\mathfrak{S}}_{g}^{*}(\Sigma), \hat{f}_{i}(\tau, \hat{z})$ are regular $q$-differentials on $S(\tau)$.

(v) These $q$-differentials $\hat{f}_{i}(\tau, \hat{z})$ are linearly independent if and only if $\tau \notin Z$.

Theorem 2. Let $g \geqq 2$ and $q \geqq 2$ be integers. Let $\tau_{0} \in \delta^{I, J} \widetilde{S}_{g}(\Sigma)$, where $g_{s}\left(\tau_{0}\right) \geqq 2$ for some $s=0,1, \cdots, 2 g-3$. Then there exist an analytic subvariety $Z \subset \mathfrak{S}_{g}^{I, J}(\Sigma)$ and $d=(2 q-1)(g-1)$ holomorphic functions $f_{s, i}(\tau, z)$, $(\tau, z) \in \sum_{t=0}^{2 g-3} \widetilde{F}_{t} \mathcal{S}_{g}^{I, J}(\Sigma)$, for each $s=0,1,2, \cdots, 2 g-3$ having the following properties (i)-(v):

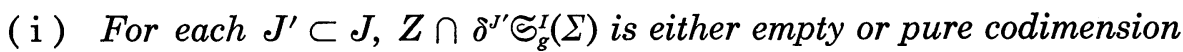
1 in $\delta^{J^{\prime}} \Im_{g}^{I}(\Sigma)$.

(ii) $\tau_{0} \notin Z$.

(iii) Suppose $\tilde{S}_{s}(\tau)=\tilde{S}_{t}(\tau)$ and $\tilde{G}_{t}(\tau)=T \tilde{G}_{s}(\tau) T^{-1}$ with $T \in$ Möb. Then

$$
f_{s, i}(\tau, z)=f_{t, i}(\tau, T(z)) T^{\prime}(z)^{q} \quad \text { for } z \in \Omega^{\prime}\left(\tilde{G}_{s}(\tau)\right), i=1,2, \cdots, d .
$$

(iv) For $i=1,2, \cdots, d$, let $\hat{f}_{i}(\tau, \hat{z})\left(\tau \in \mathfrak{S}_{g}^{I, J}(\Sigma)\right.$ and $\left.\hat{z} \in S(\tau)\right)$ be similarly defined as in Theorem 1 from $f_{s, i}(\tau, z)$. Then $\hat{f}_{i}(\tau, \hat{z})$ are regular $q$-differentials on $S(\tau)$. 
(v) These q-differentials $\hat{f}_{i}(\tau, \hat{z})$ are linearly independent if and only if $\tau \notin Z$.

8-2. Theorem 3. Let $g \geqq 2$ be an integer and let $\tau_{0} \in \hat{\subseteq}_{g}^{*}(\Sigma)$. Then there exist a neighborhood $N$ of $\tau_{0}$ in $\hat{\subseteq}_{g}^{*}(\Sigma)$ and $g$ holomorphic functions $f_{s, i}(\tau, z),(\tau, z) \in \sum_{l=0}^{2 g-3} \widetilde{F}_{t} \hat{\aleph}_{g}^{*}(\Sigma) \mid N$, for each $s=0,1, \cdots, 2 g-3$, having the following properties (i) and (ii), where the vertical segment means a restriction:

(i ) Suppose $\tilde{S}_{s}(\tau)=\tilde{S}_{t}(\tau)$ and $\tilde{G}_{t}(\tau)=T \tilde{G}_{s}(\tau) T^{-1}$ with $T \in$ Möb. Then

$$
f_{s, i}(\tau, z)=f_{t, i}(\tau, T(z)) T^{\prime}(z)^{q} \quad \text { for } z \in \Omega,\left(\tilde{G}_{s}(\tau)\right), i=1,2, \cdots, g .
$$

(ii) For $i=1,2, \cdots, g$, let $\hat{f}_{i}(\tau, \hat{z})(\tau \in N$ and $\hat{z} \in S(\tau))$ be similarly defined as in Theorem 1 . Then $\hat{f}_{i}(\tau, \hat{z})$ are linearly independent regular 1-differentials on $S(\tau)$.

This theorem is obtained from Theorems 1 and 2 by modifying the method in [2].

\section{REFERENCES}

[1] L. Bers, Spaces of degenerating Riemann surfaces, Ann. of Math. Studies, 79 (1974) , 43-55.

[2] - Automorphic forms for Schottky groups, Adv. in Math., 16 (1975), 332-361.

[ 3 ] L. R. Ford, Automorphic Functions, Chelsea, New York, 1951.

[4] H. Sato, On augmented Schottky spaces and automorphic forms, I, Nagoya Math. J., 76 (1979), 151-175.

[5] - A property of new coordinates defining augmented Schottky spaces, Nagoya Math. J., 88 (1982), 73-78.

Department of Mathematics

Faculty of Science

Shizuoka University

836, Ohya, Shizuoka

422, Japan 